\title{
The new spider genus Palindroma, featuring a novel synapomorphy for the Zodariidae (Araneae)
}

\author{
Rudy JOCQUÉ ${ }^{1, *}$ \& Arnaud HENRARD ${ }^{1,2}$ \\ ${ }^{1}$ Royal Museum for Central Africa, B-3080 Tervuren, Belgium \\ ${ }^{2}$ Earth and life Institute, Biodiversity research Center, UCL-17.07.04, Bâtiment Carnoy, Croix du Sud, \\ 5, B-1348, Louvain-la-Neuve, Belgium. Email: arnaud.henrard@gmail.com. \\ *Corresponding author: rudy.jocque@africamuseum.be \\ ${ }^{1}$ urn:1sid:zoobank.org:author:CF15016C-8CD1-4C9D-9021-44CA7DC7A5D5 \\ 2 urn:1sid:zoobank.org:author:E1B02E6E-D91C-43FE-8D8C-CD102EFEE3B4
}

\begin{abstract}
The new genus Palindroma gen. nov. is described in the Cryptothelinae, Zodariidae. Apart from the type species, $P$. morogorom gen. et sp. nov. (đㅇ), the genus contains $P$. aleykyela gen. et sp. nov. (ð), P. avonova gen. et sp. nov. (ð゚), P. sinis gen. et sp. nov. (ð) and the somewhat aberrant species $P$. obmoimiombo gen. et sp. nov. $\left({ }^{\Uparrow}\right)$. The four typical representatives of the genus are characterized by the profile of the carapace with a slight dip, the absence of precoxal sclerites and the characters of the male palp with enlarged tibia, large subtegulum and tegular retrolateral knob. The genus occurs in miombo-woodland and coastal forest in East and Central Africa and this peculiar distribution is discussed. A key to the males of the Palindroma species is provided. Specimens of Palindroma have a particularly well-developed tibial process fitting in a shallow metatarsal pouch on all legs in both sexes. Although less conspicuous in some derived taxa, the feature appears to be present in all representatives of the Zodariidae, but not in possible sister-group taxa and is thus an autapomorphy of the family.
\end{abstract}

Keywords. East Africa, Central Africa, Cryptothelinae, miombo, tibial process.

Jocqué R. \& Henrard A. 2015. The new spider genus Palindroma, featuring a novel synapomorphy for the Zodariidae (Araneae). European Journal of Taxonomy 152: 1-33. http://dx.doi.org/10.5852/ejt.2015.152

\section{Introduction}

Probably the most confusing area in the systematics of the Zodariidae is the subfamily Cryptothelinae, which has been mentioned under the name Cydrelinae until the genus Cryptothele L. Koch, 1872 was eventually incorporated into it (Jocqué \& Dippenaar-Schoeman 2007; Ramirez et al. 2014). Many species had been misplaced, mainly due to the poor definition of the genera before the revision of Jocqué (1991). As a consequence, several new genera were created to accommodate the many species that had either been misplaced or that remained to be described. The Cryptothelinae has its main distribution in southern Africa and appears to be far from completely inventoried. Some large genera (Cydrela Thorell, 1870; Psammorygma Jocqué, 1991) remain to be revised and many species have not yet been described, partly because they do not fit in one of the current genera. In the present article a new genus is created to accommodate five new species. 
The family Zodariidae can be considered as well defined. However, it remains one of the most diversified spider families: it not only has a huge size range (see Jocqué et al. 2013; Russell-Smith \& Jocqué 2015) but the variation of the habitus and of morphological characters is so vast that a superficial scan of a specimen does not always easily reveal its generic or even (sub-)familial identity. Mainly for the taxa at the base of the clade (see Jocqué 1991), there may be some doubt about their inclusion in the family. Some of them (e.g., Cyrioctea Simon, 1889) lack the typical lateral claw teeth and are incorporated in the Zodariidae solely on the base of the absence of the serrula on the endites. The new synapomorphy, a prolateral tibial process on all legs in both sexes discovered during the description of the new genus, is therefore most welcome since it corroborates the inclusion of basal taxa.

\section{Material and methods}

Specimens were observed, drawn and measured with a WILD M10 stereo microscope. Details of the female genitalia and male palps were observed with a Zeiss Stemi 2000 stereo microscope. Measurements and photographs of the habitus, details of mouthparts, detached male palps and female genitalia were taken with a Leica MZ16 using the LAS automontage software (ver. 3.8). The female genitalia were dissected and digested with pancreatin, and then immersed in $75 \%$ ethanol.

For SEM photos, specimens were dried in hexamethyldisilazane $(36 \mathrm{~h})$, gold coated and examined and photographed with a JEOL 6480 LV scanning electron microscope. Types are deposited in the Royal Museum for Central Africa, Tervuren, Belgium (MRAC) and the Zoological Museum, Natural History Museum of Denmark, University of Copenhagen (ZMUC).

All measurements are in mm. All palp illustrations are from right palps. Leg spination formulas follow Jocqué (2013).

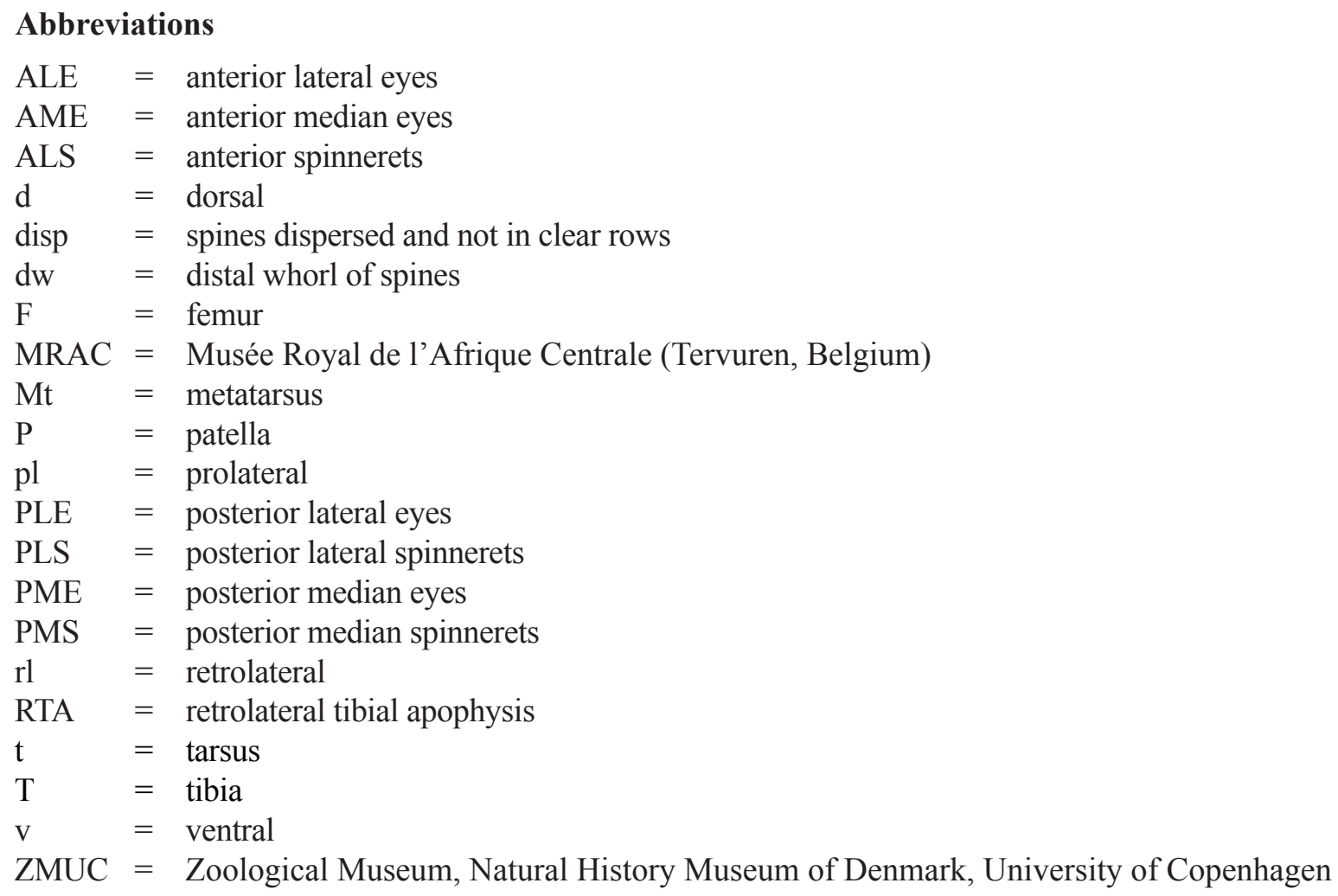


JOCQUÉ R. \& HENRARD A., Palindroma gen. nov., with novel zodariid synapomorphy

\title{
Taxonomy
}

\author{
Class Arachnida Cuvier, 1812 \\ Order Araneae Clerck, 1757 \\ Family Zodariidae Thorell, 1881 \\ Subfamily Cryptothelinae Simon, 1892 \\ Palindroma gen. nov. \\ urn:Isid:zoobank.org:act:2FF14DA8-1CEB-4F1A-941F-1DE1B368257D
}

\section{Type species}

Palindroma morogorom sp. nov.

\section{Diagnosis}

Representatives of Palindroma gen. nov. are typical sturdy Cryptothelinae with ALE in front of AME and slightly recurved to straight posterior eye row; sternum shield shaped with straight anterior margin and without precoxal sclerites; posterior legs with numerous short spines in combination with longer ventral spines; teguments of carapace, legs and abdomen often provided with patches of white or silvery setae. Male palp with large patella, large subtegulum and tegulum provided with retrolateral knob; median apophysis absent.

Palindroma gen. nov. is easily separated from Capheris Simon, 1893 and Systenoplacis Simon, 1907 by the absence of anterior concavities of the sternum. It deviates from Caesetius Simon, 1893, Psammorygma and Rotundrela Jocqué, 1999 by the absence of precoxal sclerites, from Aschema Jocqué, 1991 by the unmodified posterior legs and from Psammoduon Jocqué, 1991 by the absence of fans of supple spines on the legs; in Cydrela the sternum is slightly indented in front, the cephalic area in the carapace profile much higher and the posterior eye row more strongly recurved.

\section{Etymology}

The genus name is derived from the English term 'palindrome' originating from the Greek ' $\pi \alpha \lambda \iota v \delta \rho \circ \mu \circ \varsigma$ ', that refers to words or sentences that are identical whether they are read from front to back or the other way round. All species names of the new species described here are palindromes. The gender of the species name is feminine.

\section{Description}

Medium size spiders $(7.5-10 \mathrm{~mm})$ with reticulated to roughly granulate teguments. Carapace longer than wide ( $\mathrm{L} / \mathrm{W}<1.43-1.71)$, slightly protruding anteriorly, with silvery hairs in male, almost hairless in females apart from a few longer hairs on clypeus; widest at level of coxae II-III, narrowed to about $0.48-0.62$ times maximum width in males and 0.62-0.71 times maximum width in females (cephalic width measured on posterior tangent of PME). Cervical grooves poorly indicated. Profile flat or domed, highest at level of first coxae and with slight dip at level of fovea.

Colour: carapace medium to dark brown; chelicerae, legs, mouthparts and sternum medium to orange brown; abdomen dorsum grey with one to seven pale spots and four reddish apodemes, sides and venter white to pale grey; in males sclerotized in front of epigastric fold.

Eyes in three rows: ALE in front of AME and further apart, posterior row straight or slightly recurved and eyes far apart. All eyes subequal, AME dark, other eyes pale. Clypeus straight, slightly slanting back, height 2.5 to 4 times diameter of ALE, sometimes with some long dispersed setae. 
Chilum double, about or slightly more than twice as wide as high, lateral margin poorly defined; without setae. Chelicerae slightly conical, inner margin almost straight, with many evenly dispersed setae; with distomesal membranous lamina (Fig. 1A-B); fangs shorter than wide at base. Labium inverted Ushaped, with slightly narrowed base. Endites roughly triangular, converging, with basolateral extension accommodating palpal coxae. Sternum shield-shaped, as wide as long, without triangular extensions or precoxal sclerites; anterior margin straight, lateral margins slightly sinuous.

Legs: robust. Formula 4123 or 4132 . Spination reduced on legs I and II, well developed on III and IV. Most spines short and thick except dorsal ones on F and ventral ones on T and Mt. Patella III and IV with retrolateral boss at base of short spine. Anterior tarsi fusiform in male, usually longer than metatarsi, unmodified in female. One dorsal hinged hair on tibiae and metatarsi I and II (Fig. 1D). Trochanters with anterior concavity. Prolateral tibial process strongly developed on all legs in both sexes (see Fig. 17A-E). Three tarsal caws, paired ones with numerous teeth (Fig. 1C).

Female palp with numerous prolateral spines and some retrolateral ones (Fig. 1E); palpal claw with some small teeth at base (Fig. 1F); turned inward over less than $45^{\circ}$; without distal patch of chemosensitive setae.

Abdomen oval, with ventral row of small sclerotized apodemes; tracheal spiracle fairly small, somewhat advanced and provided with small rectangular scutellum. Both exes with six spinnerets. ALS large, conical, biarticulate. PLS and PMS provided with 1 and 3 cylindrical gland spigots, respectively. Colulus represented by haired field.

Male palp: complexity of palps very variable; patella larger than tibia (Fig. 2A), sometimes modified; RTA sometimes very small, in other species well developed. Cymbium with prolateral spines; without distal claw; sometimes with basal swelling(s); subtegulum strongly developed, sclerotized and visible in unexpanded palp; tegulum with retrolateral boss (Fig. 2B), variable in shape; embolus short, flat, distal part with flange.

Epigyne with central longitudinal depression. Spermathecae large, far apart; copulatory ducts with thick walls (Fig. 2C-F).

\section{Note}

The species Palindroma obmoimiombo gen. et sp. nov. is only tentatively and probably temporarily incorporated in the genus. It lacks several of the specific generic characters: the indented profile of the carapace, the membranous lamina of the chelicerae, the enlarged male palpal patella. On the other hand, it has a field with spinules on the flat mesal surface of the chelicerae. The latter character approaches conditions in Caesetius but that genus has a high domed carapace, precoxal sclerites and a much more strongly recurved posterior eye row. The species apparently belongs to an as yet undescribed genus but with only one species and in the absence of females we have refrained from creating a new genus for it.

\section{Distribution}

Palindroma is found in forest and miombo regions of central and eastern Africa in the Democratic Republic of the Congo, Malawi and Tanzania.

\section{Key to males}

1. Chelicerae almost rectangular as viewed in front, with distomesal extension well developed, without mesal field of spinules; carapace domed, with slight dip at level of fovea; palp with swollen patella, tibia with one RTA or RTA poorly developed 
JOCQUÉ R. \& HENRARD A., Palindroma gen. nov., with novel zodariid synapomorphy

- Chelicerae conical, with distomesal extension, inconspicuous, with mesal field of spinules; carapace profile flat, without dip; palp with unmodified patella, with two RTA ......P. obmoimiombo sp. nov.

2. Carapace profile with slight dip at level of fovea; anterior tarsi with same length as metatarsi or

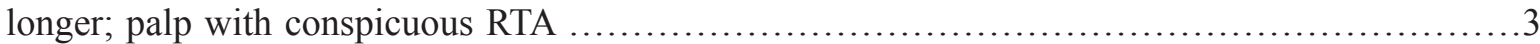

- Carapace profile with marked dip at level of fovea; anterior tarsi shorter than metatarsi; palp with

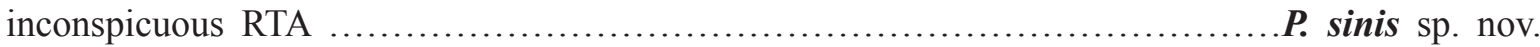

3. Anterior tarsi clearly longer than metatarsi; tegulum with well-developed basal or retrolateral

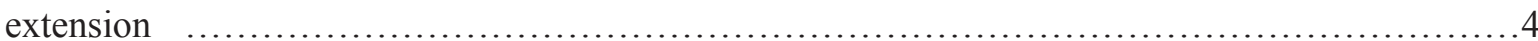

- Anterior tarsi only slightly longer than metatarsi; tegulum with longitudinal ventral ridge ending medially in poorly developed knob

.P. avonova sp. nov.

4. RTA thin, long and strongly tapered apically; tegulum sub-apically with retrolateral knob

P. morogorom sp. nov.

- RTA broad, short, with strongly sclerotized rounded extremity; tegulum basally with welldeveloped conical extension P. aleykyela sp. nov.

Palindroma morogorom gen. et sp. nov. urn:1sid:zoobank.org:act:20ACA48F-42F0-403C-A81A-21425B87484C

Figs 1A-F, 2A-D, 3A-G, 4A-D, 5A-C, 16, 17A-E

\section{Diagnosis}

The male of $P$. morogorom gen. et sp. nov. can be recognized by the long, strongly tapered RTA. The female has a characteristic epigyne with central depression showing two dark areas, flanked by a kidneyshaped area on either side and the inverted funnel-shaped pattern in front.

\section{Etymology}

The species name is an arbitrary combination of letters forming a noun in apposition and containing 'morogoro', the region where the types were found.

\section{Material examined}

Holotype

TANZANIA: $\widehat{\text { }}$, Uzungwa Mts, Morogoro Region, Mwanihana Forest, 744'29.2” S, 3653'17.7” E, 1800-1850 m, 25-29 Sep.1984, montane rain forest, pitfall trap, N. Scharff (ZMUC).

\section{Paratypes}

TANZANIA: $2 \hat{\partial}, 1 \hat{\phi}$, together with holotype; $4 \hat{\partial} \hat{\partial}$, as previous; $1 \hat{\partial}$, as previous; $1 \hat{\partial}$, as previous; $4 \hat{\partial} \hat{\partial}$, as previous; $5 \hat{\partial} \hat{\partial}$, as previous (2 $\hat{\delta} \hat{\partial}$ in MRAC 244094); 1 q, 28-29 Sep. 1984, further as

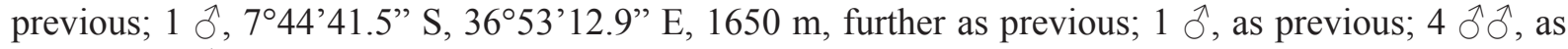
previous; 1 o, $1650 \mathrm{~m}, 25-29 \mathrm{Sep}$. 1984, further as previous.

\section{Other material}

TANZANIA: $3 \hat{\jmath} \widehat{\partial}$, Uzungwa Mts, Iringa Region, Mufundi-Kigogo Forest Reserve, $8^{\circ} 40^{\prime} 00.5^{\prime \prime} \mathrm{S}$, $35^{\circ} 12^{\prime} 35.2^{\prime \prime}$ E, $1900 \mathrm{~m}, 7-15$ Oct. 1984, montane rain forest, pitfall trap, N. Scharff (ZMUC); 2 đ̊ ô, as previous; $3 \hat{\partial} \hat{\partial}$, as previous; 1 + $, 1700 \mathrm{~m}, 5-10$ Oct. 1984, further as previous; $1 \hat{\partial}$, as previous; $1 \hat{\partial}$, $8^{\circ} 40^{\prime} 53.5^{\prime \prime} \mathrm{S}, 35^{\circ} 14^{\prime} 59.1$ ” E, $1700 \mathrm{~m}, 8-10$ Oct. 1984, further as previous; 1 ㅇ, Uzungwa Mts, Iringa Region, Uzungwa Scarp Forest above Chita village, $8^{\circ} 40^{\prime} 53.5^{\prime \prime} \mathrm{S}, 35^{\circ} 12^{\prime} 35.2^{\prime \prime}$ E, $750 \mathrm{~m}, 23$ Oct. -14 Nov. 1984, lowland rain forest, N. Scharff (ZMUC); 1 ग, 1600-1650 m, 8-13 Nov. 1984, montane rain forest, further as previous; 1 O, 1 \&, Iringa district, Uzungwa scarp Forest Reserve, $11 \mathrm{~km}$ E of 
Masisiwe village, Kihanga stream, 1800 m, 8²2’05.7” S, 355'41.6” E, 17-27 May 1997, ZMUC; 1 ㅇ, Uluguru Mts, Bunduki, Moy Mgeta, $7^{\circ} 20^{\prime}$ S, 37³8’ E, 30 Apr.-2 May 1957, P. Basilewsky \& N. Leleup (MRAC 111892).

\section{Note}

Georeferences of the collections made in 1984 are approximations.
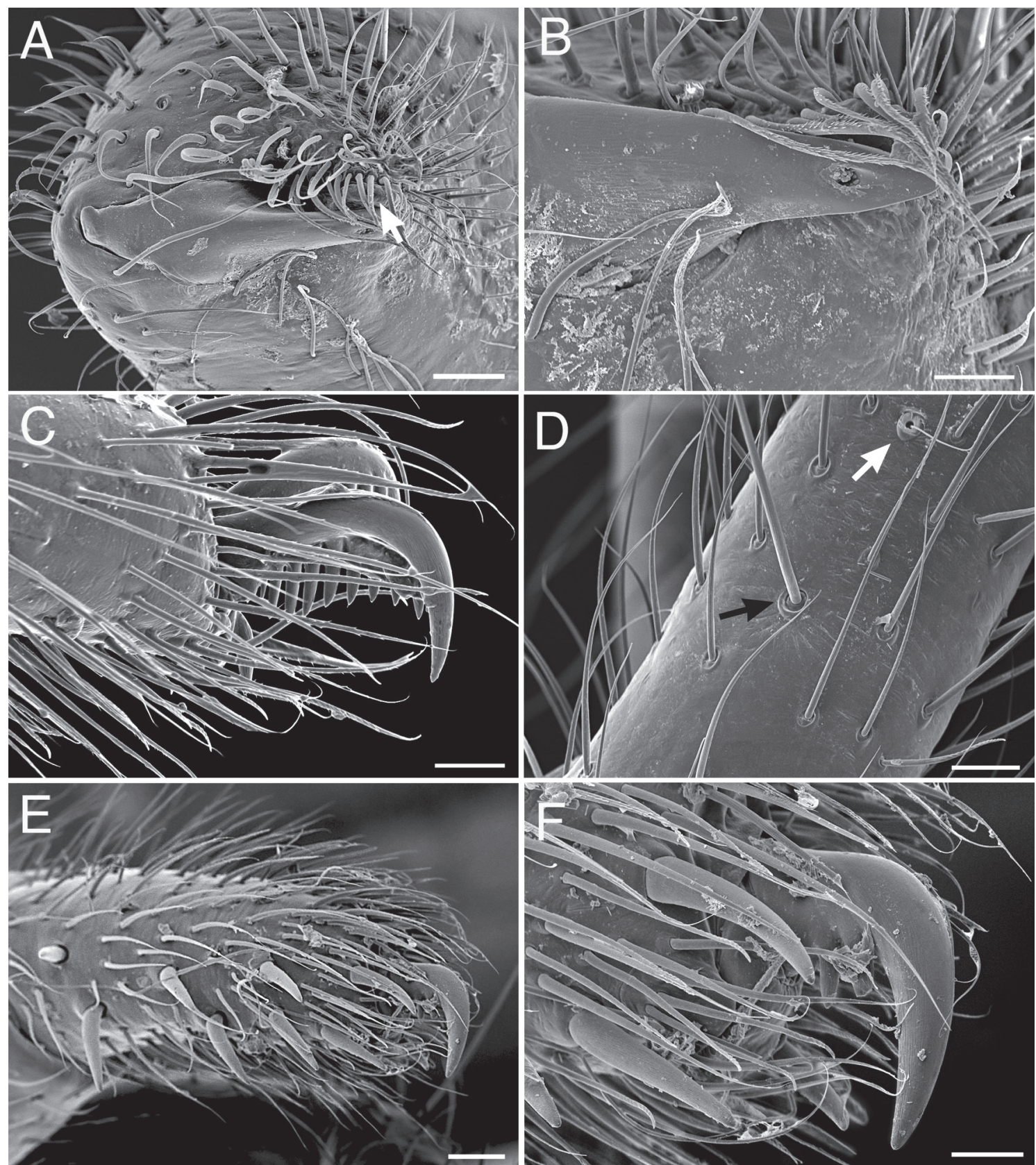

Fig. 1. Palindroma morogorom gen. et sp. nov. Scanning electron miocrographs. A-D. $\widehat{o}$ from Kigogo Reserve. E-F. Paratype 9 . A. Right chelicera, ventral view, arrow shows membranous lamina. B. As previous, detail. C. Leg I, tarsal claws, lateral view. D. Tibia I, hinged hair (black arrow), trichobothrium (white arrow). E. o right palp, lateral view. F. Detail of previous. Scale bars: A, E-F $=100 \mathrm{~mm}, \mathrm{~B}-\mathrm{D}=$ $50 \mathrm{~mm}$. 
JOCQUÉ R. \& HENRARD A., Palindroma gen. nov., with novel zodariid synapomorphy

\section{Description}

Male (holotype)

Total length 8.02; carapace 4.05 long, 2.77 wide, narrowed to 1.35 in eye region.
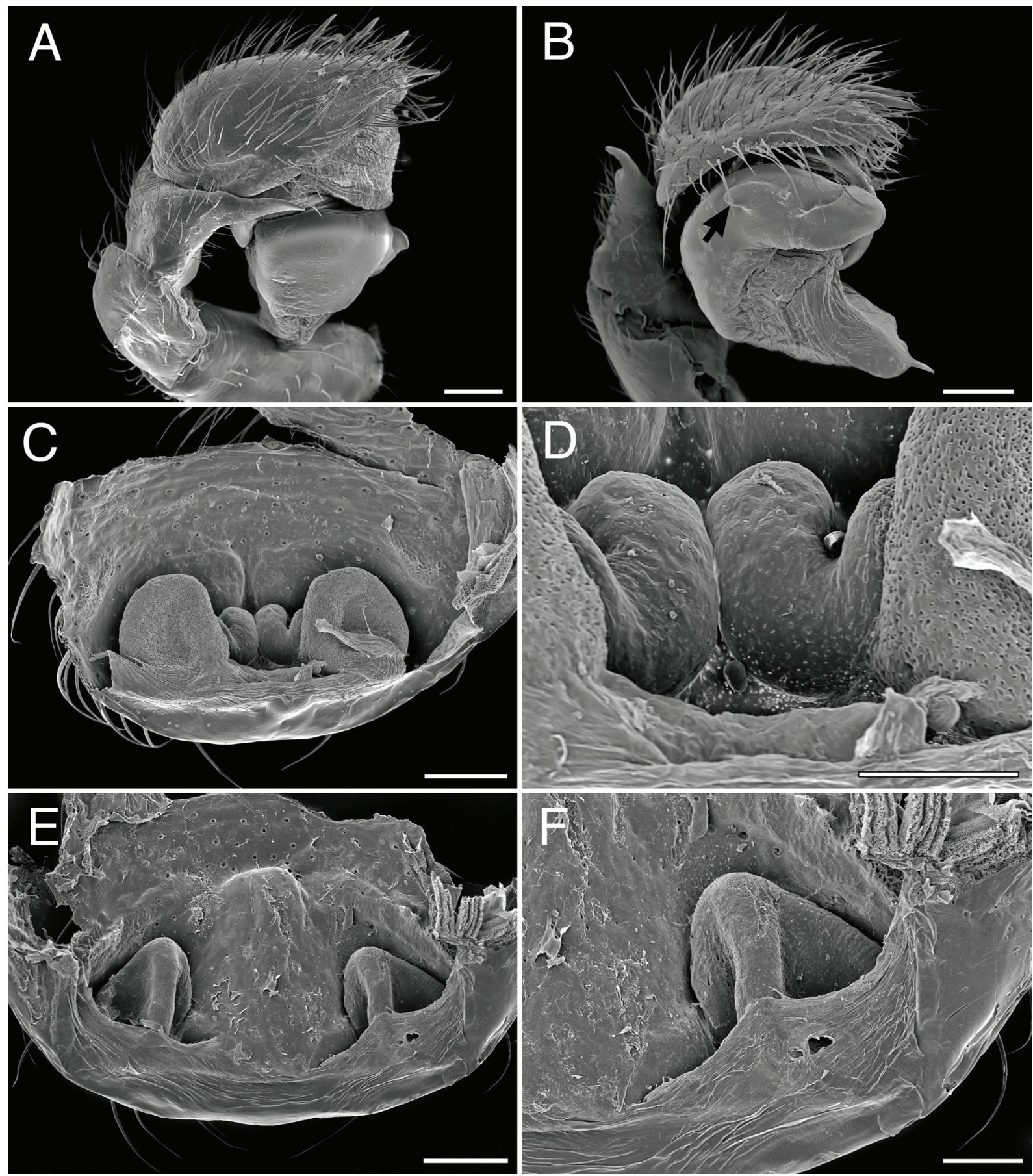

Fig. 2. - A-D. Palindroma morogorom gen. et sp. nov. Scanning electron miocrographs. A-B. $\widehat{\delta}$ from Kigogo Reserve. C-D. $\rightarrow$ from Uzungwa Scarp Forest Reserve, paratype. A. ô palp, expanded, retrolateral view. B. Idem, anterolateral view. Arrow shows tegular retrolateral boss. C. Epigyne, digested, dorsal view. D. Idem, detail. - E-F. Palindroma avonova gen. et sp. nov., paratype, ․ . E. Epigyne, digested, dorsal view F. Idem, detail. Scale bars: A-C, E = $200 \mathrm{~mm}, \mathrm{D}, \mathrm{F}=100 \mathrm{~mm}$. 
Colour: carapace and chelicerae medium brown without silvery setae (Fig. 3A, C-D); legs and sternum brownish orange (Fig. 3B); abdomen dorsum grey with pair of adjacent pale spots in front, a pair of pale spots in the middle followed by white bar and spot in front of spinnerets; four reddish apodemes in frontal half clearly delimited and two above the pedicel (Fig. 3A); sides grey with well delimited frontal apodemes; venter grey with lateral rows of apodemes and two rows of small apodemes behind well developed brownish epiandrum. Tracheal spiracle with small rectangular scutum followed by white membranous area in front of yellowish brown spinnerets.

Carapace granulated, cervical grooves slightly smoother (Fig. 3A). Clypeus with dispersed setae.

Eyes: AME: 0.12; ALE: 0.12; ALE-ALE: 0.21; AME-ALE: 0.16; PME: 0.12; PLE: 0.15; PME-PME: 0.13; PME-PLE: 0.31. Clypeus 0.54 or 4.5 times width of ALE.

Chilum double, each sclerite 0.10 high, 0.33 wide; no setae. Sternum shield-shaped, 1.42 long, 1.35 wide.

Legs: anterior tarsi longer than metatarsi.
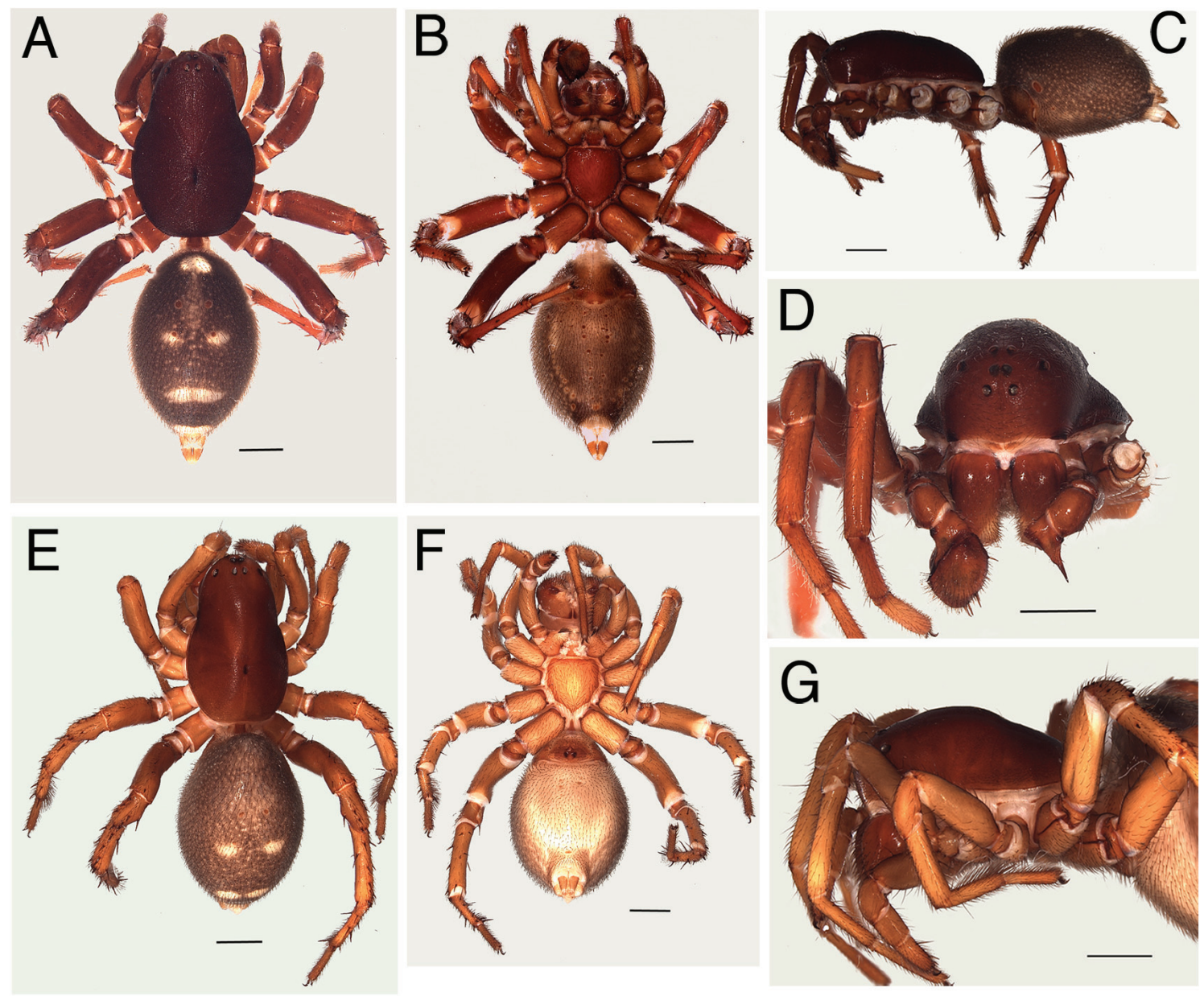

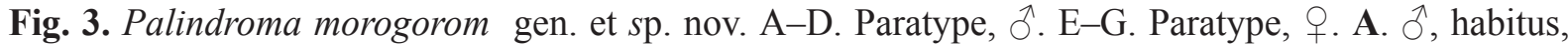
dorsal view. B. Idem, ventral view. C. Idem, lateral view. D. Idem, frontal view. E. ㅇ, habitus, dorsal view. F. Idem, ventral view. G. Idem, lateral view. Scale bars $=1 \mathrm{~mm}$. 
JOCQUÉ R. \& HENRARD A., Palindroma gen. nov., with novel zodariid synapomorphy

\begin{tabular}{ccccc}
\hline Spination & & & & \\
& F & P & T & Mt \\
\hline I & $\mathrm{d} 3$ & - & pl1 & $\mathrm{dw} 3$ \\
II & $\mathrm{d} 3$ & - & pl1 & $\mathrm{dw} 2$ \\
III & $\mathrm{d} 3$ & pl3d2rl1 & pl2d45r11v3 & d1dw5 \\
IV & $\mathrm{d} 1$ & pl3d3rl1 & pl2d5rl21v2 & 5dispdw5
\end{tabular}
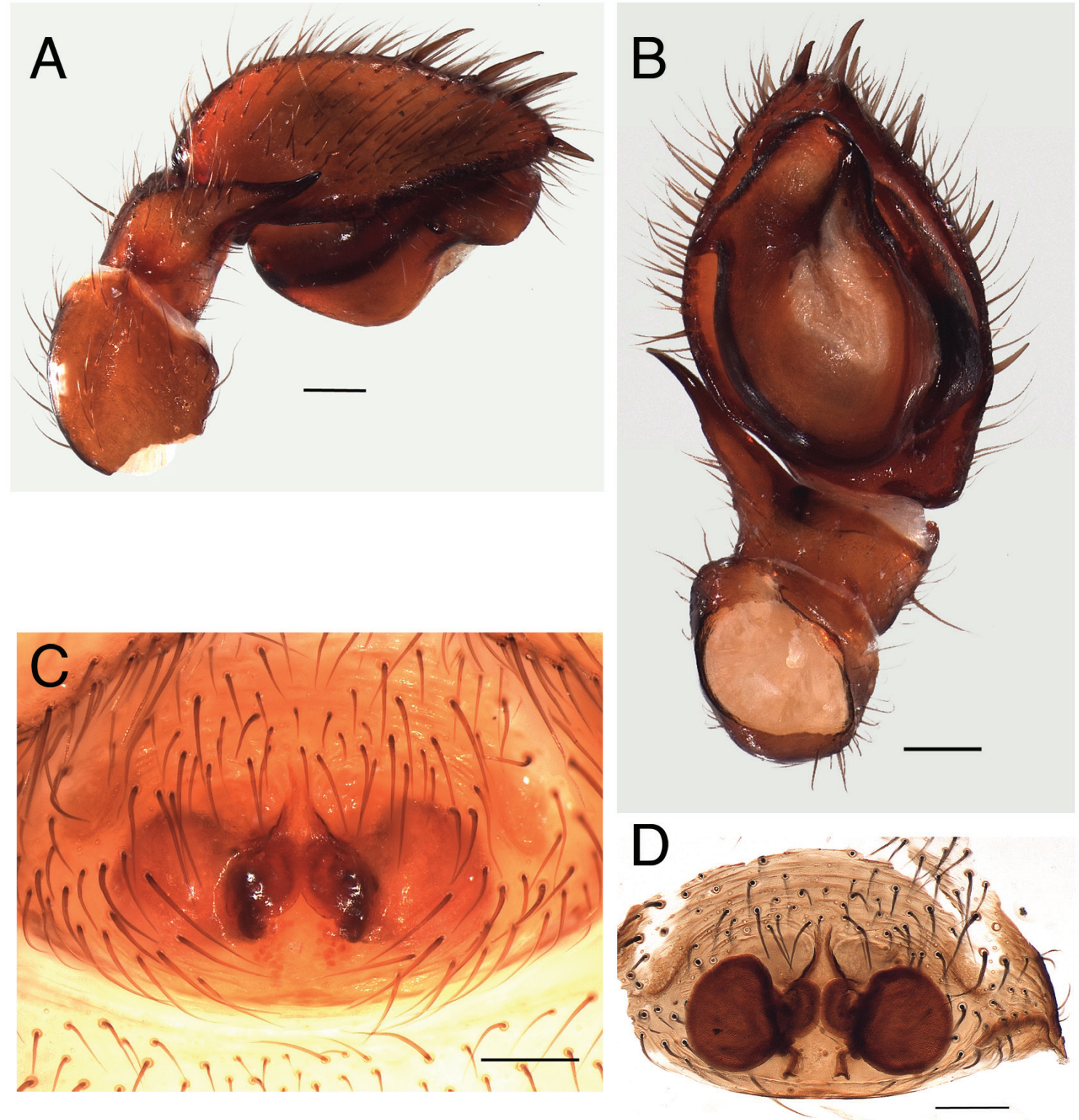

Fig. 4. Palindroma morogorom gen. et sp. nov. A-B. Paratype, ô. C-D. Paratype, ․ . A. $\widehat{\partial}$, palp, retrolateral view. B. Idem, ventral view. C. + , epigyne, ventral view. D. Idem, cleared in methylsalicylate, dorsal view. Scale bars $=0.2 \mathrm{~mm}$. 
Leg measurements

\begin{tabular}{ccccccc} 
& F & P & T & Mt & t & Total \\
\hline I & 2.10 & 0.91 & 1.61 & 1.33 & 1.40 & 7.35 \\
II & 1.82 & 0.91 & 1.26 & 1.19 & 0.98 & 6.16 \\
III & 1.89 & 1.05 & 1.26 & 1.26 & 0.91 & 6.37 \\
IV & 2.24 & 1.05 & 1.89 & 2.17 & 1.26 & 8.61
\end{tabular}

Male palp (Figs 2A-B, 4A-B, 5A-B): RTA with broad base strongly tapered to sharp extremity turned upward and outward; cymbium with several spines in distal half; subtegulum strongly sclerotized; tegulum with retrolateral knob; embolus originating on prolateral distal part of tegulum, broad at base, strongly tapered to sinuous sharp tip.

Female (paratype collected with holotype)

Total length 9.30; carapace 4.12 long, 2.41 wide, narrowed to 1.70 in eye region.

Colour: carapace chestnut brown with few silvery setae (Fig. 3E-G); chelicerae medium brown, sternum and legs orange brown; abdomen as in male but apodemes less well marked.

Carapace smooth. Clypeus with dispersed setae.

Eyes: AME: 0.13; ALE: 0.13; ALE-ALE: 0.31; AME-ALE: 0.23; PME: 0.15; PLE: 0.18; PME-PME: 0.14; PME-PLE: 0.38 . Clypeus 0.59 or 4.5 times width of ALE.
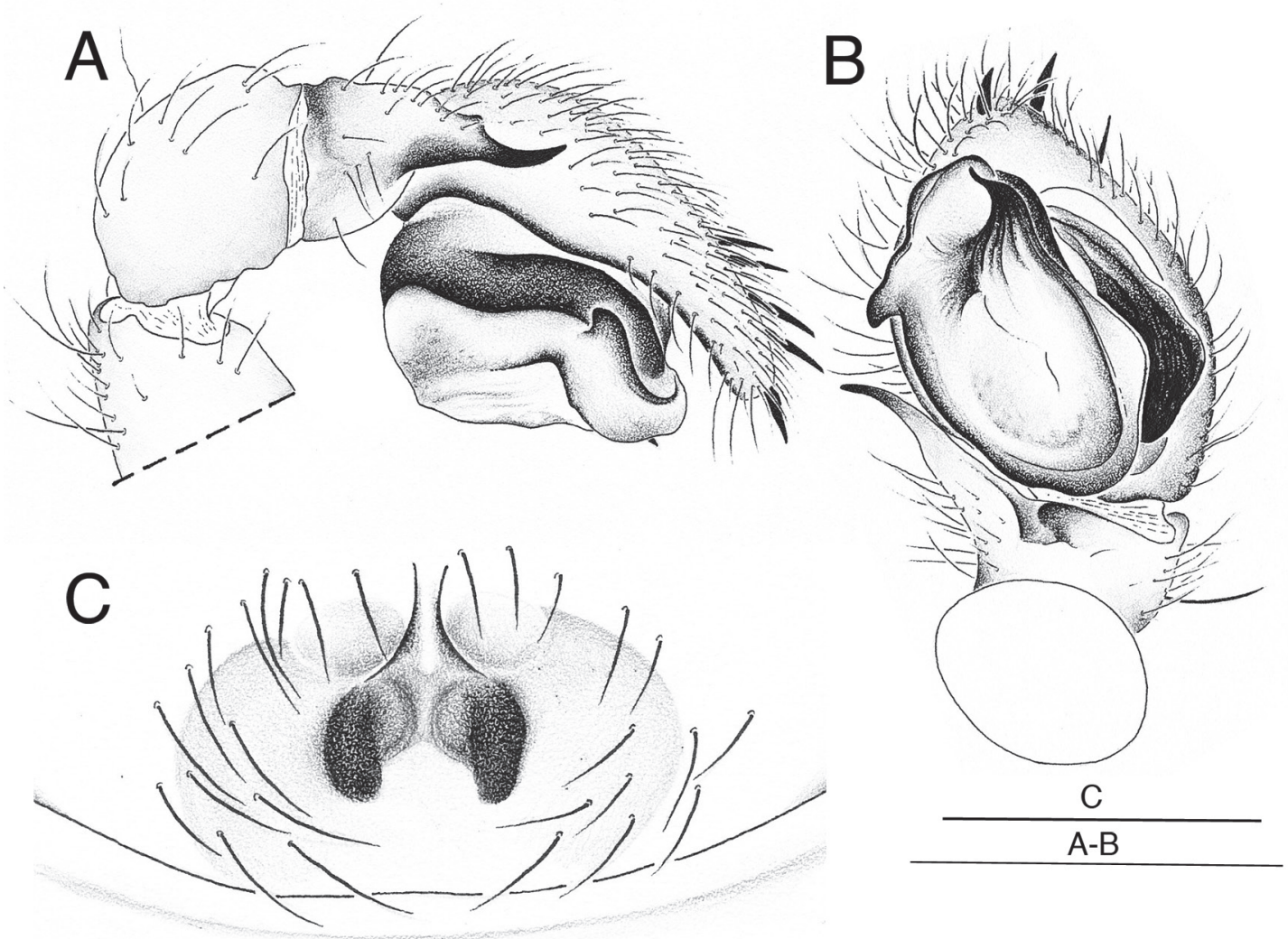

Fig. 5. Palindroma morogorom gen. et sp. nov. A-B. Holotype, $\overbrace{}^{\lambda}$. C. Paratype,, . A. $\overbrace{}^{\lambda}$, palp, retrolateral view. B. Idem, ventral view. C. + , epigyne, ventral view. Scale bars: $A-B=1 \mathrm{~mm}, \mathrm{C}=0.5 \mathrm{~mm}$. 
JOCQUÉ R. \& HENRARD A., Palindroma gen. nov., with novel zodariid synapomorphy

Chilum double, each sclerite 0.10 high, 0.33 wide; no setae. Sternum shield-shaped, 1.42 long, 1.42 wide.

Legs: Anterior tarsi not modified, shorter than metatarsi.

Spination

\begin{tabular}{ccccc} 
& F & P & T & Mt \\
\hline I & d3 & - & pl1 & pl1 \\
II & d3 & - & - & dw3 \\
III & d3 & pl3d3rl1 & pl2d4rl1v2-2-1 & d1r11dw5 \\
IV & d1 & pl4d3rl1 & pl2d5rl2v2-2-1 & 6disp dw5
\end{tabular}

Leg measurements

\begin{tabular}{ccccccc} 
& F & P & T & Mt & t & Total \\
\hline I & 2.31 & 0.98 & 1.75 & 1.33 & 1.26 & 7.63 \\
II & 1.54 & 0.91 & 1.40 & 1.19 & 1.05 & 6.09 \\
III & 1.89 & 1.05 & 1.26 & 1.47 & 1.05 & 6.72 \\
IV & 2.31 & 1.19 & 1.96 & 2.31 & 1.26 & 9.03
\end{tabular}

Palp with pectinated claw turned inward over $45^{\circ}$. Tarsus provided with eight retrolateral spines.

Epigyne (Figs 2C-D, 4C-D, 5C): central depression with two dark areas; large kidney-shaped area on either side; inverted funnel-shaped pattern in front.

\section{Distribution}

Known from the Uzungwa and Uluguru Mts. in Tanzania (Fig. 16).

Palindroma aleykyela gen. et sp. nov. urn:1sid:zoobank.org:act:D41FD227-07BF-4D3C-AA5D-C2571D88E741

Figs $6 \mathrm{~A}-\mathrm{D}, 7 \mathrm{~A}-\mathrm{B}, 16$

\section{Diagnosis}

The males of $P$. aleykyela gen. et sp. nov. are easily recognized by large palpal patella and the conspicuous basal extension of the tegulum.

\section{Etymology}

The species name is an arbitrary combination of letters forming a noun in apposition and containing 'Kyela', the name of the type locality.

\section{Material examined}

\section{Holotype}

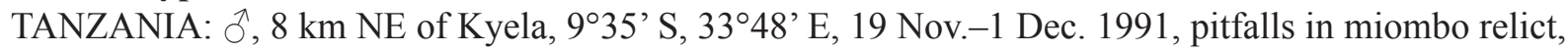
R. Jocqué (MRAC 173610).

\section{Other material}

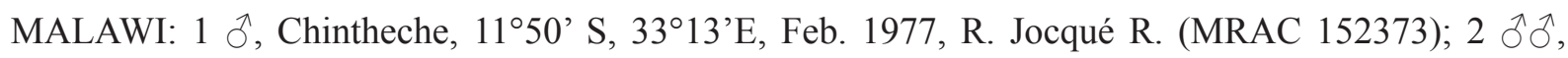

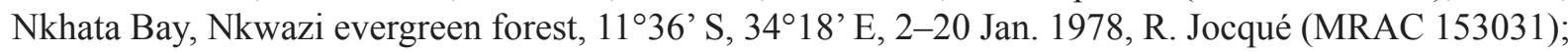

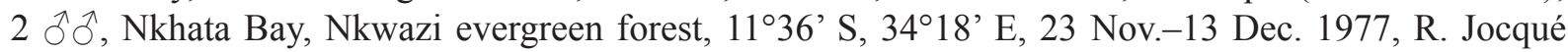


(MRAC 153248); 1 $\delta^{1}$, Nyika Plateau, near entrance gate on the Chelinda-Rumphi road, $10^{\circ} 40^{\prime} \mathrm{S}$, 3350' E, 3-22 Dec. 1981, secondary Brachystegia woodland with Uapaca, pitfalls, R. Jocqué (MRAC 156005); 1 ô, as previous (MRAC 156064).

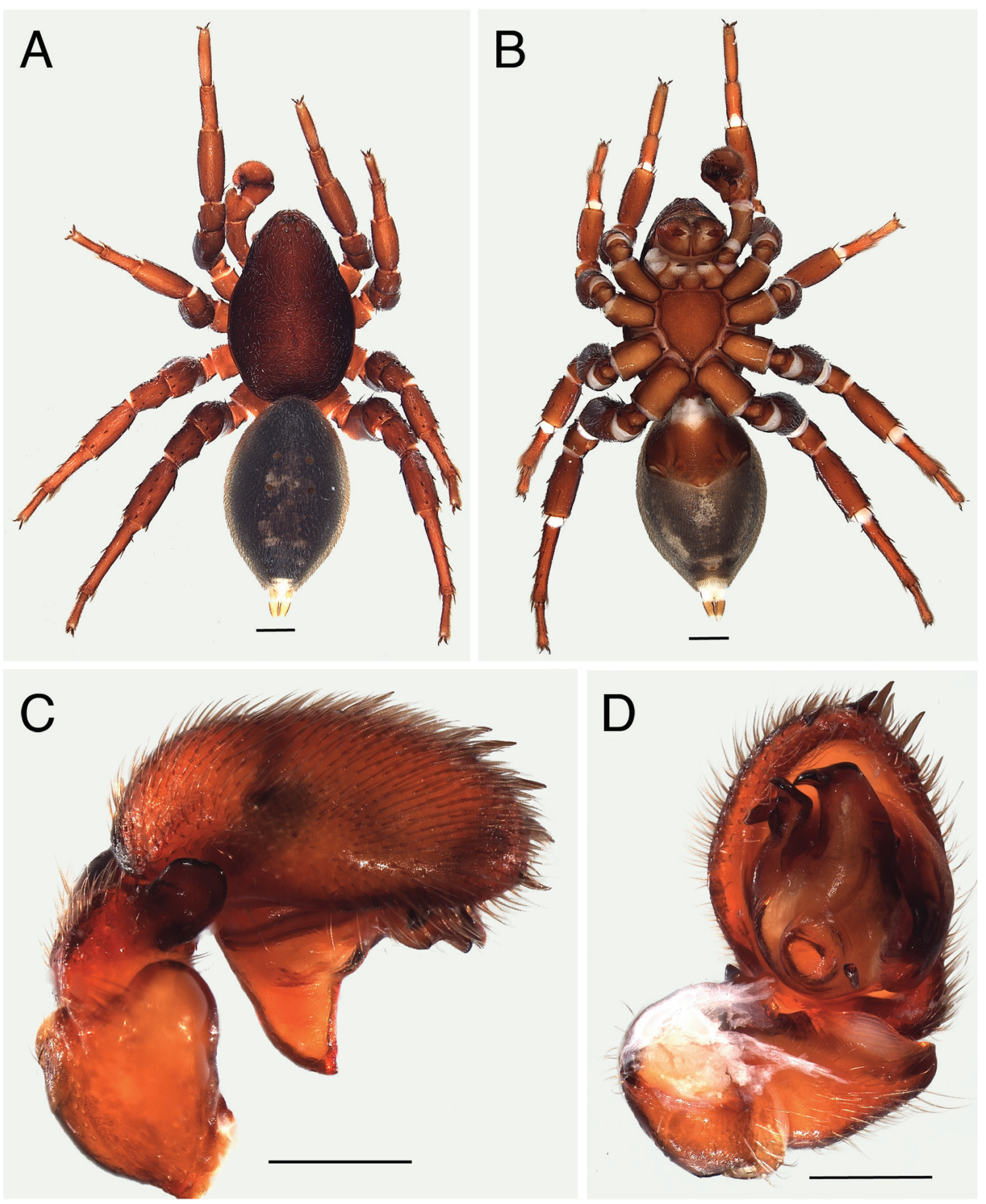

Fig. 6. Palindroma aleykyela gen. et sp. nov., holotype, ठ̂. A. Habitus, dorsal view. B. Idem, ventral view. C. Palp, retrolateral view. D. Idem, ventral view. Scale bars: A-B $=1 \mathrm{~mm}, \mathrm{C}-\mathrm{D}=0.5 \mathrm{~mm}$. 
JOCQUÉ R. \& HENRARD A., Palindroma gen. nov., with novel zodariid synapomorphy

\section{Description}

Male (holotype)

Total length 9.87; carapace 4.97 long, 2.98 wide, narrowed to 1.42 in eye region.

Colour: carapace uniform dark brown with dispersed silvery hairs (Fig. 6A-B); chelicerae sternum and legs brownish orange; abdomen: dorsum grey with two pairs of reddish apodemes, white dispersed short setae, denser in posterior half, white spot in front of yellow spinnerets; sides grey with well delimited frontal reddish apodeme; venter pale grey, sclerotized and orange in front of epigastric fold.

Carapace coarsely granulate. Clypeus without erect setae.

Eyes: AME: 0.16; ALE: 0.20; ALE-ALE: 0.21; AME-ALE: 0.07; PME: 0.12; PLE: 0.18; PME-PME: 0.15; PME-PLE: 0.41. Clypeus 0.62 or 3.1 times width of ALE.

Chilum double, each sclerite 0.08 high, 0.16 wide; no setae. Sternum shield-shaped with sinuous sides, 2.00 long, 1.70 wide.

Chelicerae with mesodistal membranous extension. Endites swollen at base.

Legs: anterior tarsi fusiform, longer than metatarsi.

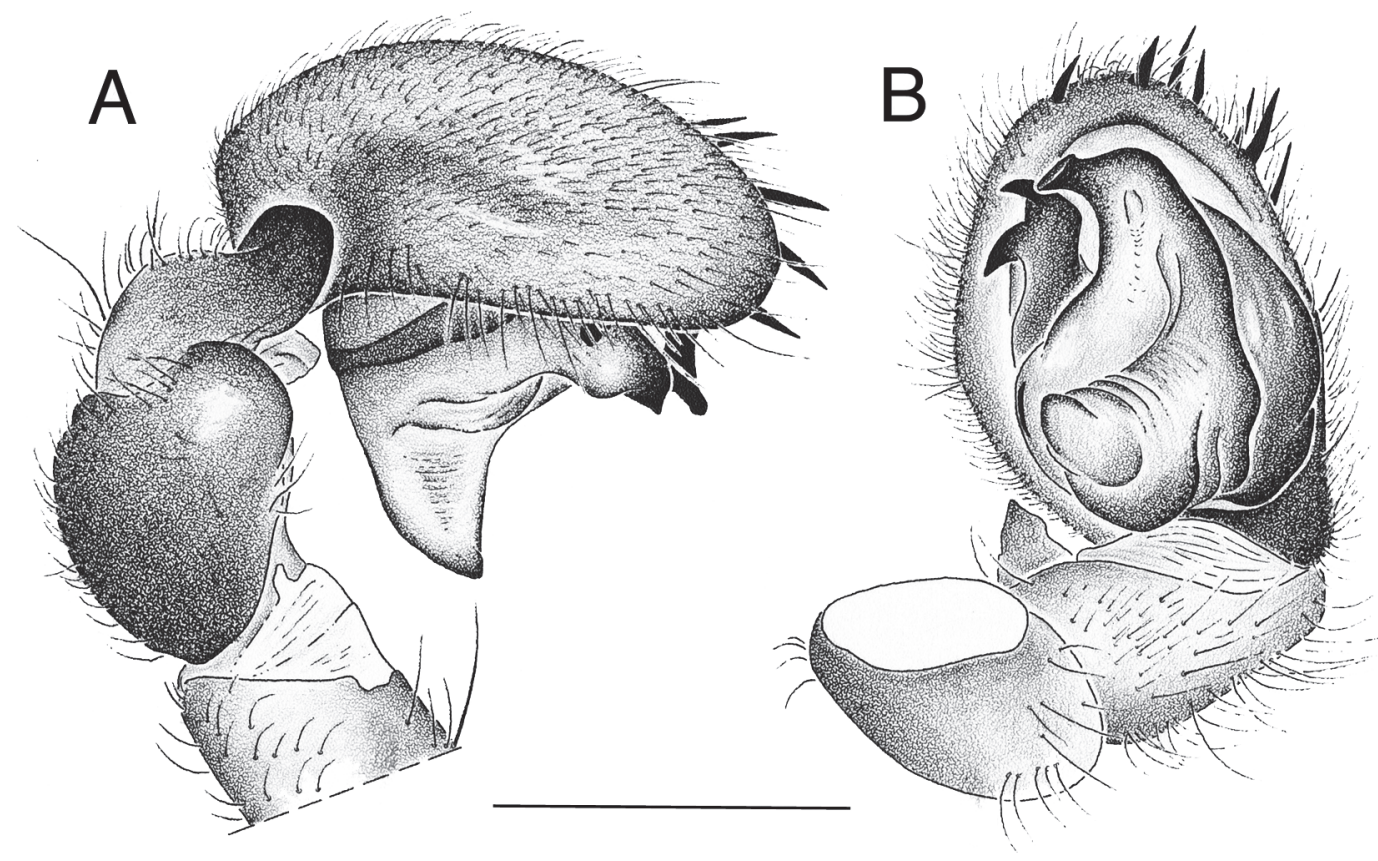

Fig. 7. Palindroma aleykyela gen. et sp. nov., $\widehat{\partial}$ (MRAC 153248). A. Palp, retrolateral view. B. Idem, ventral view. Scale bar $=1 \mathrm{~mm}$. 
Spination

\begin{tabular}{ccccc} 
& F & P & T & Mt \\
\hline I & d2 & - & pl1v1-1-2 & dw3 \\
II & d2 & - & v1-1-2 & dw2 \\
III & d1-3 & pl2d3rl1 & pl3d4rl2v1-1-2 & 8dispdw6 \\
IV & d1-3 & pl2d3rl1 & pl3d5rl2v1-1-2 & 10dispdw5
\end{tabular}

Leg measurements

\begin{tabular}{ccccccc} 
& F & P & T & Mt & t & Total \\
\hline I & 2.38 & 1.19 & 1.96 & 1.26 & 1.40 & 8.19 \\
II & 2.17 & 1.19 & 1.68 & 1.12 & 1.05 & 7.21 \\
III & 2.31 & 1.33 & 1.47 & 1.47 & 1.05 & 7.63 \\
IV & 2.73 & 1.47 & 2.03 & 2.38 & 1.33 & 9.94
\end{tabular}

Epiandrum well developed, with two small, white, membranous spots.

Male palp (Figs 6C-D, 7A-B): patella large, with conspicuous mesolateral bulge; tibia with prolateral dorsal protrusion; RTA broad, short, with strongly sclerotized, rounded extremity; base strongly tapered to sharp extremity turned upward and outward; cymbium with seven prolateral spines in distal half; subtegulum strongly sclerotized; tegulum with large, pointed, basal extension, distally with short, dark, hooked process (HP); embolus short, curved, with rounded process at base.

\section{Female}

Unknown.

\section{Distribution}

Known from miombo woodland in northern Malawi and northern Tanzania (Fig. 16).

Palindroma avonova gen. et sp. nov. urn:Isid:zoobank.org:act:E54283D6-495A-4859-B5D0-84887059EBA2

Figs 8A-D, 9A-C, 10A-D, 16

\section{Diagnosis}

The male of $P$. avonova gen. et sp. nov. is easily recognized by the characters of the palp: the swollen patella, the thick RTA, the ridged tegulum and the sickle shaped embolus. The female has a characteristic epigyne with longitudinal central depression separated from the posterior margin by a rectangular plate.

\section{Etymology}

The species name is an arbitrary combination of letters forming a noun in apposition and containing 'nova' (new) as the genus as well as the species are new.

\section{Material examined}

\section{Holotype}

TANZANIA: ${ }^{\lambda}$, Muheza district, Manga Forest Reserve, 502’ S, 3847’ E, Oct.-Dec.1994, Frontier Tanzania (ZMUC). 


\section{Paratypes}

TANZANIA: $1 \hat{\partial}$, together with holotype (MRAC 244092); $2 \hat{\jmath}, 1 \stackrel{\rho}{\circ}$, together with holotype (ZMUC);

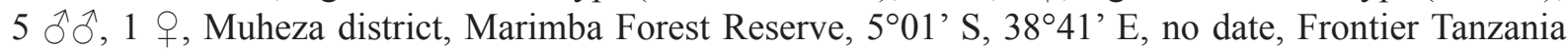
(ZMUC).
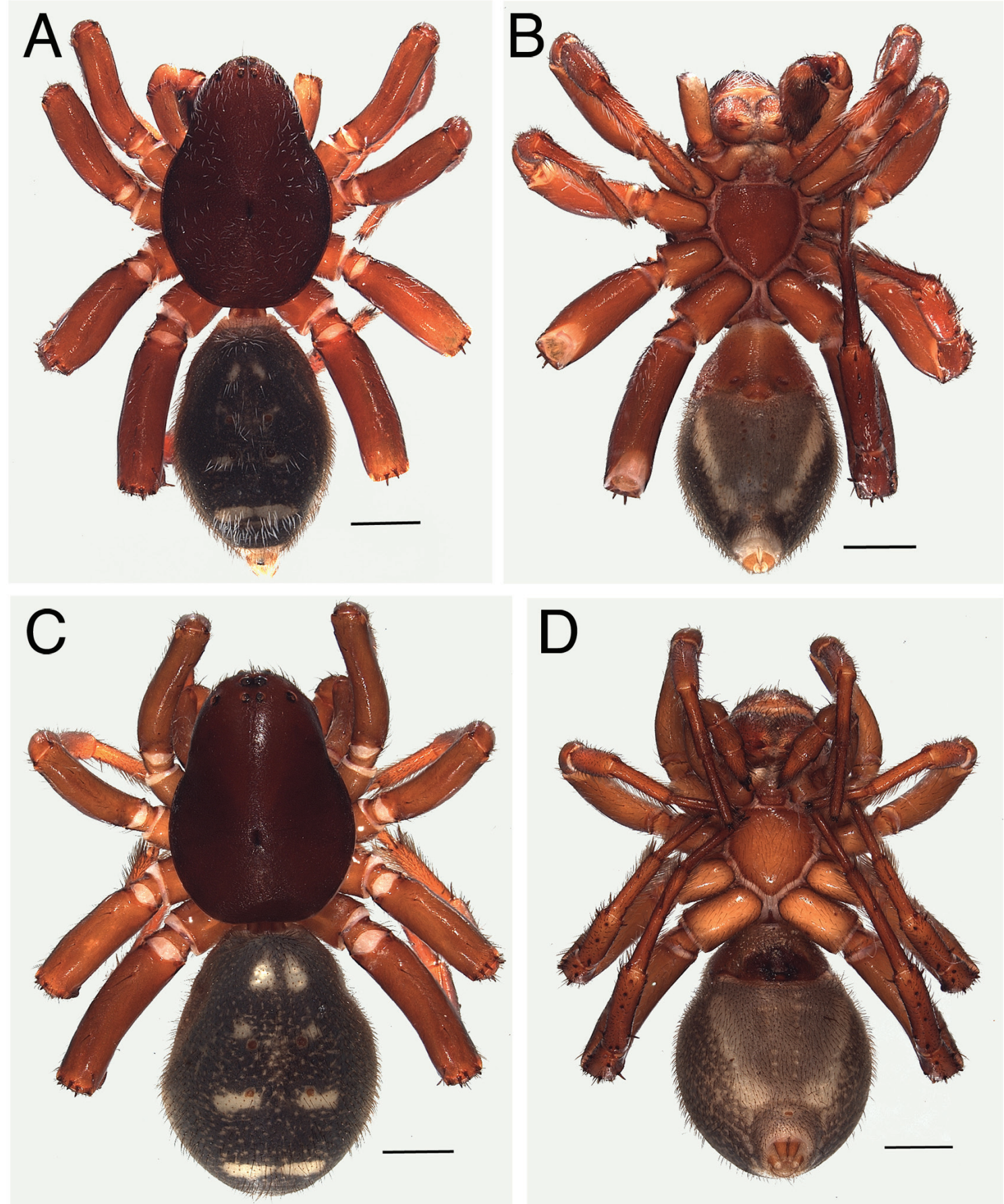

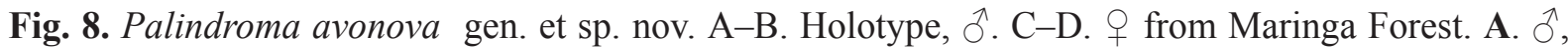

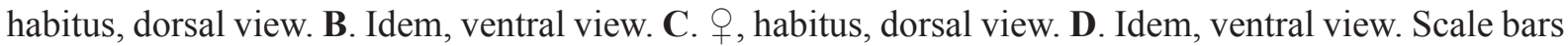
$=1 \mathrm{~mm}$. 


\section{Other material}

TANZANIA: 1 옹, Coastal Region, Kazimzumbwi Forest Reserve, 657’ S, 39³’ E, Jan.-Feb. 1991,

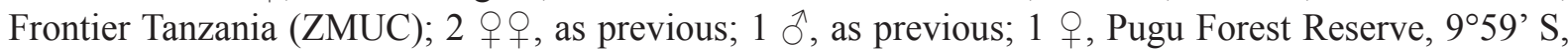
397’ E, 25 Apr. 1981, leaf litter, K.M. Howell (MRAC 159097).

\section{Description}

Male (holotype)

Total length 7.60; carapace 3.83 long, 2.63 wide, narrowed to 1.28 in eye region.

Colour: Carapace dark brown with few silvery setae (Fig. 8A-B); chelicerae, sternum and legs medium brown; Mt I, II with white hairs. Abdomen: dorsum with white setae, denser in front and on posterior white bar; three pairs of white spots, one pair of adjacent white bars and a white bar in front of spinnerets; four apodemes well developed; sides dark grey; venter pale grey, well separated from sides by oblique white band on either side; sclerotized in front of epigastric fold; four rows of apodemes; spinnerets yellowish brown.

Carapace granulated.
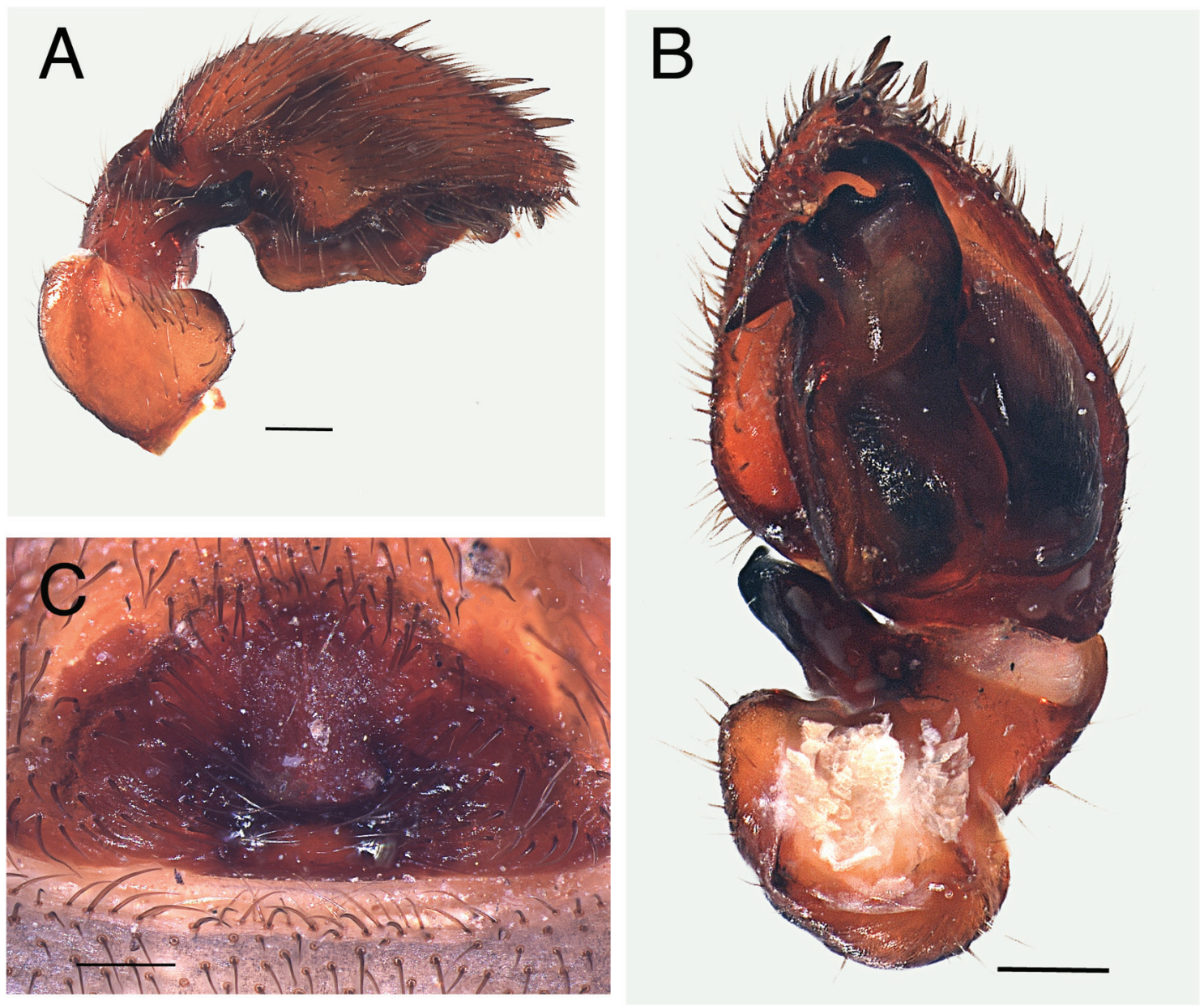

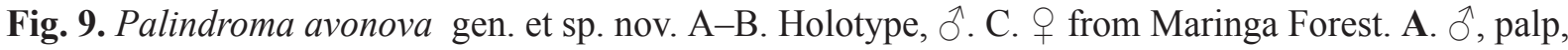
retrolateral view. B. Idem, ventral view. C. , epigyne, ventral view. Scale bars $=0.2 \mathrm{~mm}$. 
JOCQUÉ R. \& HENRARD A., Palindroma gen. nov., with novel zodariid synapomorphy

Eyes: AME: 0.10; ALE: 0.14; ALE-ALE: 0.15; AME-ALE: 0.13; PME: 0.08; PLE: 0.13; PME-PME: 0.12; PME-PLE: 0.30. Clypeus 0.39 or 2.8 times width of ALE.

Chilum double, each sclerite 0.13 high, 0.20 wide; no setae. Chelicerae with distomesal membranous swelling. Sternum shield-shaped, 1.42 long, 1.28 wide.

Legs: anterior tarsi slightly longer than metatarsi.

Spination

\begin{tabular}{ccccc} 
& F & P & T & Mt \\
\hline I & $\mathrm{d} 3$ & - & - & $\mathrm{dw} 2$ \\
II & $\mathrm{d} 3$ & - & v3 & $\mathrm{dw} 2$ \\
III & $\mathrm{d} 3+2^{*}$ & pl3d1-2rl1 & pl2d3rl1v2-2-2 & d1dw5 \\
IV & $\mathrm{d} 3+3^{*}$ & pl3d1-2rl1 & pl3d5rl2v1-1-2 & 4dispdw5
\end{tabular}

* 3 long, slender setae +2 or 3 short, thick, distal setae

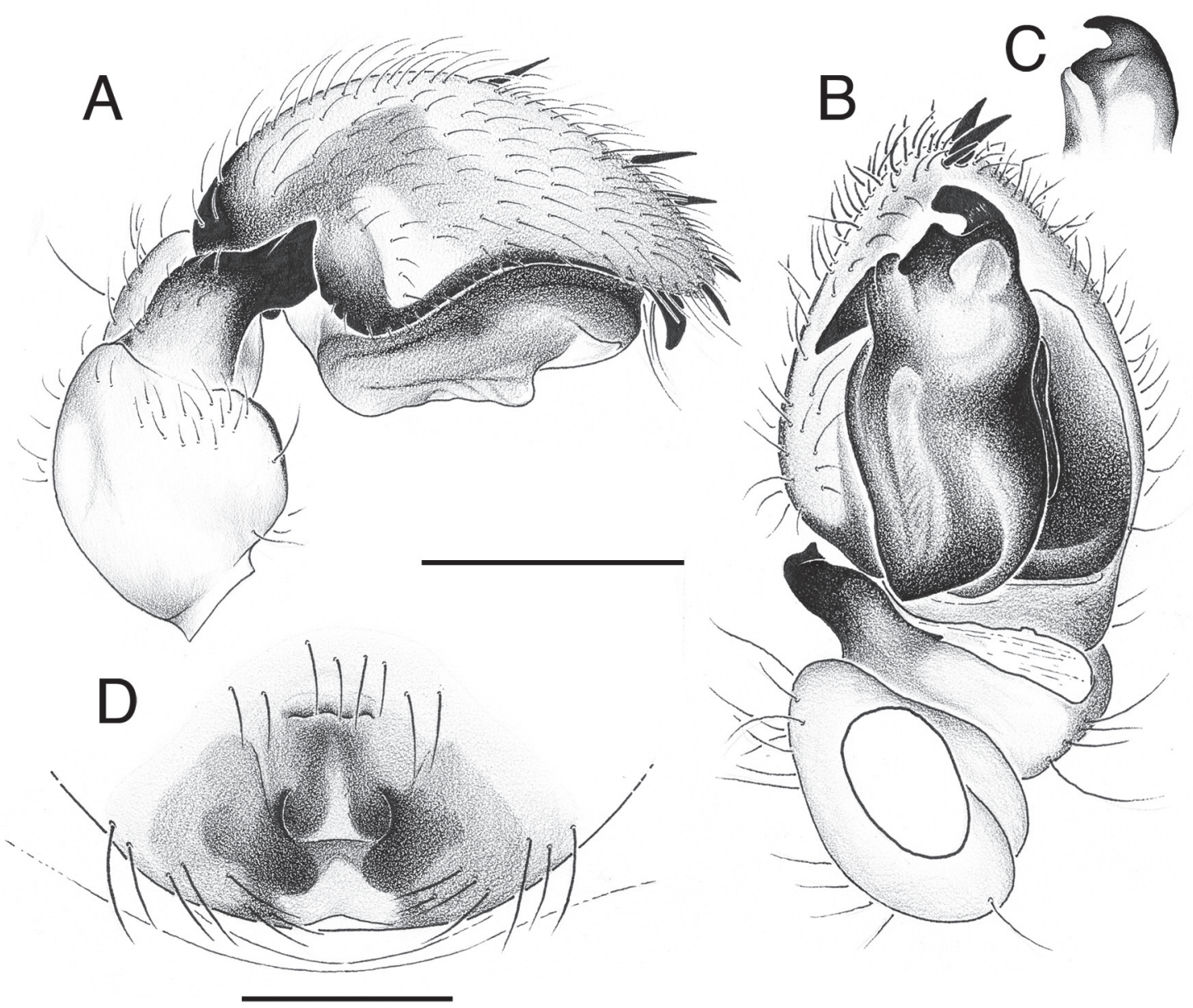

Fig. 10. Palindroma avonova gen. et sp. nov. A-B. Holotype, $\widehat{\partial}$. C. Paratype, $\widehat{\partial}$, from Marimba Forest. D. $q$ from Maringa forest. A. $O^{\top}$, palp, retrolateral view. B. Idem, ventral view. C. Palp detail of paratype, enbolus, ventral view. D. + , epigyne, ventral view. Scale bars $=0.5 \mathrm{~mm}$. 
Leg measurements

\begin{tabular}{ccccccc} 
& F & P & T & Mt & t & Total \\
\hline I & 1.68 & 0.84 & 1.54 & 0.98 & 1.05 & 6.09 \\
II & 1.61 & 0.84 & 1.26 & 0.98 & 0.70 & 5.39 \\
III & 1.75 & 0.84 & 1.33 & 1.12 & 0.70 & 5.74 \\
IV & 2.24 & 1.12 & 1.75 & 2.03 & 0.98 & 8.12
\end{tabular}

Male palp (Figs 9A-B, 10A-C): patella large, swollen; tibia with short, rounded dorsal process; RTA strongly sclerotized, short, thick, with truncated tip; cymbium with several spines in distal half; with basal swellings near dorsal tibial process and RTA; tegulum with longitudinal ventral ridge ending medially in poorly developed knob; embolus short, sickle shaped; MA strongly sclerotized, conical, pointing backward; subtegulum large, strongly sclerotized.

Female (paratype from Marimba Forest)

Total length 8.02; carapace 3.76 long, 2.63 wide, narrowed to 1.63 in eye region.

Colour: carapace dark brown without silvery setae (Fig. 8C-D); chelicerae, sternum and legs medium brown without white setae; abdomen: pattern as in male but without white setae and not sclerotized in front of epigastric fold but for epigyne. Further as in male.

Carapace: finely reticulated. Clypeus with dispersed setae.

Eyes: AME: 0.18; ALE: 0.20; ALE-ALE: 0.18; AME-ALE: 0.20; PME: 0.13; PLE: 0.16; PME-PME: 0.10; PME-PLE: 0.31. Clypeus 0.43 or 2.2 times width of ALE.

Chilum double, each sclerite 0.10 high, 0.26 wide; no setae. Sternum shield-shaped, 1.42 times as long as wide.

Legs:

Spination

\begin{tabular}{ccccc} 
& F & P & T & Mt \\
\hline I & $\mathrm{d} 3$ & - & - & $\mathrm{dw} 2$ \\
II & $\mathrm{d} 3$ & - & - & $\mathrm{dw} 2$ \\
III & $\mathrm{d} 2+1^{*}$ & pl3d3 & pl2d3rl1v1-1-1 & d1dw5 \\
IV & $\mathrm{d} 2+3^{*}$ & pl3d3rl1 & pl5d2rl2v1-1-1 & 4dispdw5
\end{tabular}

* 2 long, slender setae +1 or 3 short, thick, distal setae

Leg measurements

\begin{tabular}{ccccccc} 
& F & P & T & Mt & t & Total \\
\hline I & 1.26 & 0.91 & 1.68 & 1.12 & 1.05 & 6.02 \\
II & 1.82 & 0.77 & 1.33 & 0.98 & 0.91 & 5.81 \\
III & 1.82 & 0.98 & 1.12 & 1.40 & 1.05 & 6.37 \\
IV & 2.45 & 1.05 & 1.96 & 2.10 & 1.19 & 8.75
\end{tabular}

Palp: claw with three teeth, turned inward over $45^{\circ}$. Tarsus provided with strong retrolateral spines.

Epigyne (Figs 9C, 10D): dark, fairly large central depression longitudinal, separated from posterior margin by transverse rectangular plate. 
JOCQUÉ R. \& HENRARD A., Palindroma gen. nov., with novel zodariid synapomorphy

\section{Distribution}

Known from forest in Muheza District in Tanzania (Fig. 16).

Palindroma obmoimiombo gen. et sp. nov.

urn:lsid:zoobank.org:act:F5095BED-45CF-4480-B925-F86B7795A9B4

Figs $11 \mathrm{~A}-\mathrm{E}, 12 \mathrm{~A}-\mathrm{D}, 13 \mathrm{~A}-\mathrm{B}, 16$

\section{Diagnosis}

The male of $P$. obmoimiombo gen. et sp. nov. is easily recognized by its colour, the inconspicuous cheliceral lamina and the characters of the palp: the unmodified patella, the presence of a dorsolateral tibial apophysis

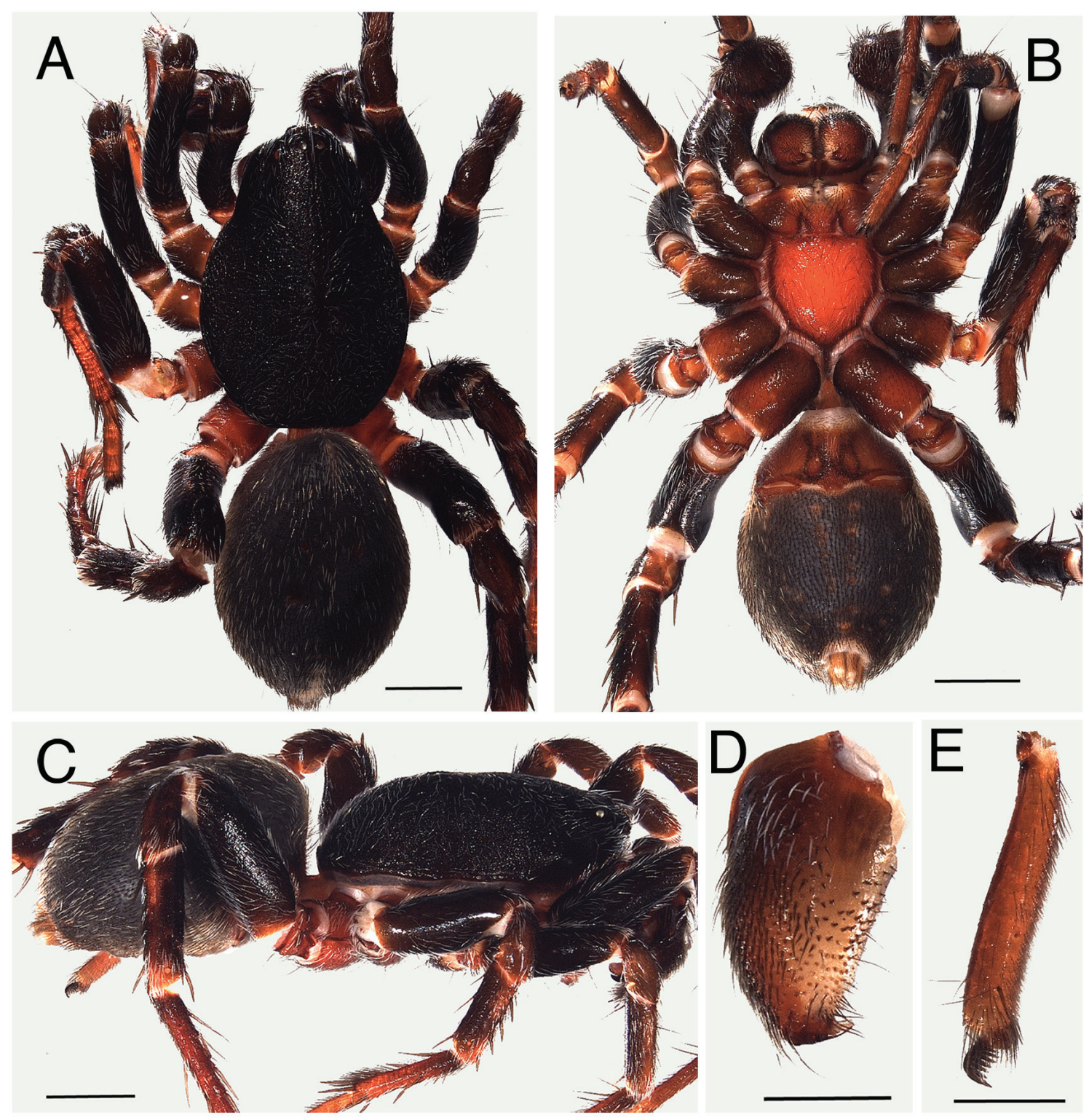

Fig. 11. Palindroma obmoimiombo gen. et sp. nov., paratype, $\widehat{\partial}$ (MRAC 241634). A. Habitus, dorsal view. B. Idem, ventral view. C. Idem, lateral view. D. Left chelicera, ventral view, showing mesal field of spinules. E. Tarsus 4, retrolateral view. Scale bars: A-C $=1 \mathrm{~mm}, \mathrm{D}-\mathrm{E}=0.5 \mathrm{~mm}$ ). 
and a bifid RTA, the large subtegulum with ridges and posterior swelling and the thin embolus lying in a groove of the tegulum.

\section{Etymology}

The species name is an arbitrary combination of letters forming a noun in apposition and containing 'miombo', the type of woodland in which the type material was collected.

\section{Material examined}

\section{Holotype}

DEMOCRATIC REPUBLIC OF THE CONGO: ${ }^{\wedge}$, Mikembo Sanctuary near Lubumbashi, $11^{\circ} 28^{\prime}$ S, 27³9’ E, 8 Nov. 2010, 1180 m, miombo woodland, by hand, R. Jocqué (MRAC 241633).

\section{Paratype}

DEMOCRATIC REPUBLIC OF THE CONGO: 1 đ , Mikembo Sanctuary near Lubumbashi, $11^{\circ} 28^{\prime}$ S, 27³9' E, 1-31 Oct. 2010, 1180 m, miombo woodland, by hand, Cemdika \& Jenny (MRAC 241634).
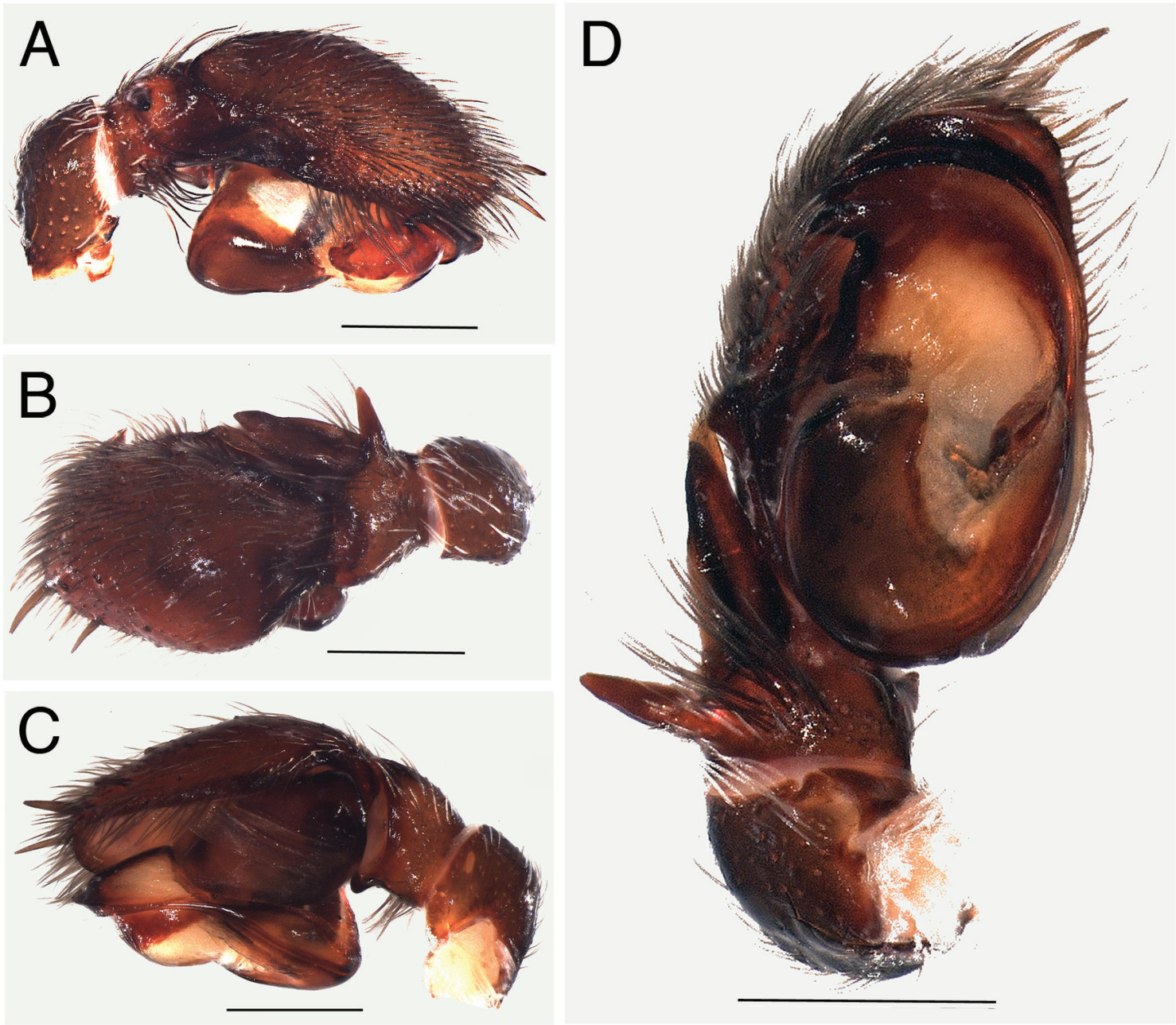

Fig. 12. Palindroma obmoimiombo gen. et sp. nov., holotype, $\widehat{\partial}$ (MRAC 241633). A. Palp, retrolateral view. B. Idem, dorsal view. C. Idem, prolateral view. D. Idem, ventral view. Scale bars $=0.5 \mathrm{~mm}$. 
JOCQUÉ R. \& HENRARD A., Palindroma gen. nov., with novel zodariid synapomorphy

\section{Other material}

None.

\section{Description}

Male (holotype)

Total length 7.46; carapace 3.91 long, 2.84 wide, narrowed to 1.56 in eye region.

Colour: carapace black with numerous silvery hairs (Fig. 11A-C); chelicerae dark brown with white setae in proximal half, medium brown with dark setae in distal half and white distomesal part (Fig. 11D); sternum orange; coxae and trochanters brownish orange, femora dark brown to black with numerous white setae; patellae and tibiae dark brown with few white setae; metatarsi and tarsi medium brown (Fig. 11E); abdomen dark grey with silvery setae, four reddish apodemes, three anastomosing pale spots in front of brownish spinnerets; sides dark grey with few silvery setae and reddish frontal apodeme; venter dark grey with two rows of small apodemes and a few near sides; orange in front of epigastric fold.

Carapace granulated, profile almost flat.

Eyes: AME: 0.13; ALE: 0.15; ALE-ALE: 0.31; AME-ALE: 0.08; PME: 0.13; PLE: 0.12; PME-PME: 0.10; PME-PLE: 0.25 . Clypeus 0.64 or 4.3 times width of ALE.

Chilum double, each sclerite 0.10 high, 0.20 wide; no setae. Chelicerae with mesal side flat, with short spines; distomesal membranous swelling, absent. Sternum shield-shaped, 1.49 long, 1.42 wide, produced between posterior coxae.

Legs: anterior tarsi shorter than metatarsi.

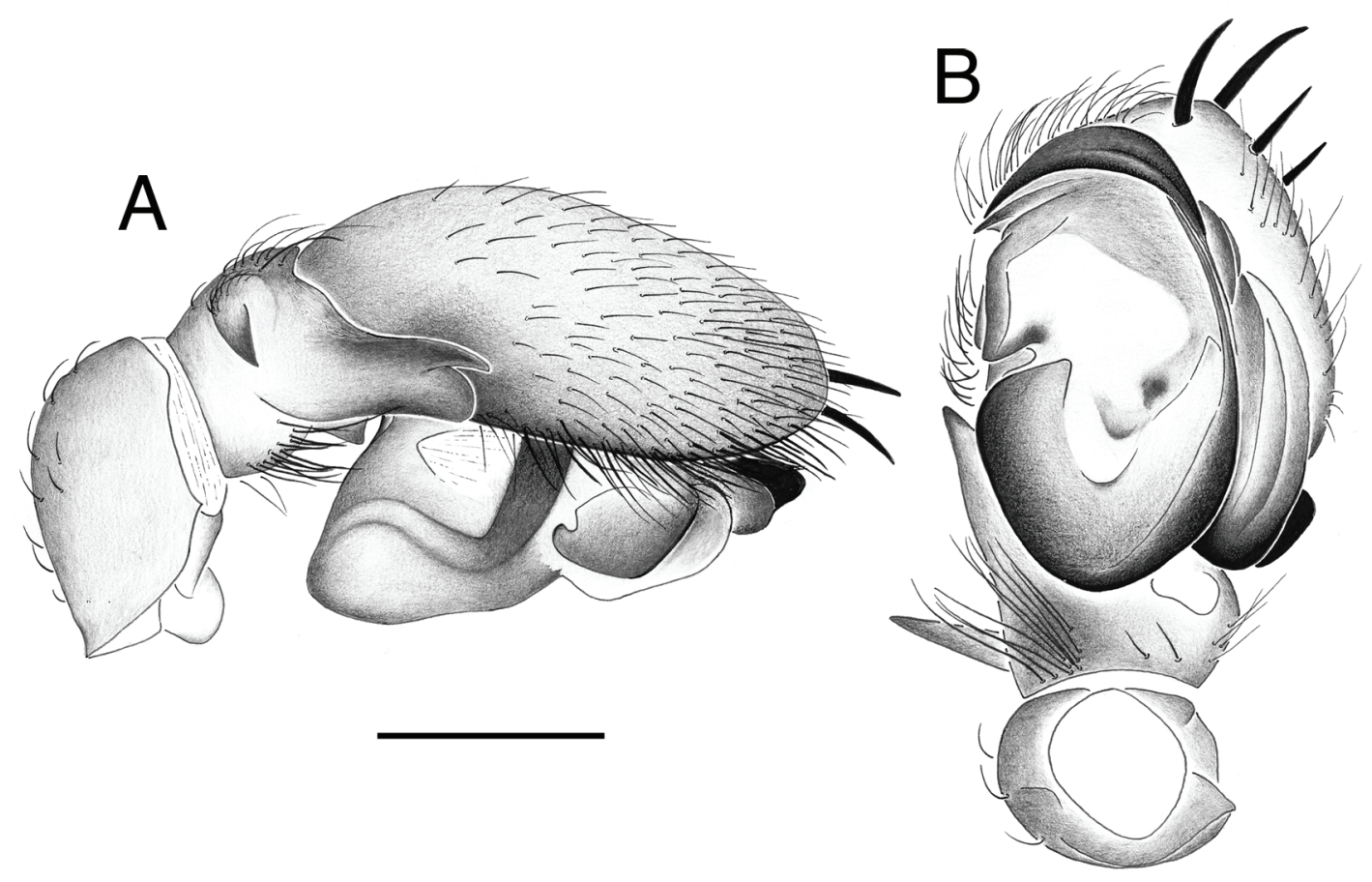

Fig. 13. Palindroma obmoimiombo gen. et sp. nov., holotype, $\widehat{o}$ (MRAC 241633). A. Palp, retrolateral view. B. Idem, dorsal view. Scale bars $=0.5 \mathrm{~mm}$. 
Spination

\begin{tabular}{ccccc} 
& F & P & T & Mt \\
\hline I & d1 & - & pl2 & v2-2 \\
II & d1 & - & pl2 & v2dw3 \\
III & pl1d3 & pl3d1rl1 & pl3d3rl2v1-2-2 & 8disp dw5 \\
IV & pl1d3 & pl1d1r11 & pl3d3rl2v1-1-2 & 11disp dw5
\end{tabular}

Leg measurements

\begin{tabular}{ccccccc} 
& F & P & T & Mt & t & Total \\
\hline I & 2.17 & 0.98 & 1.96 & 1.47 & 1.26 & 7.84 \\
II & 1.89 & 0.91 & 1.33 & 1.40 & 1.12 & 6.65 \\
III & 1.68 & 1.05 & 1.26 & 1.61 & 1.26 & 6.86 \\
IV & 2.24 & 1.19 & 1.75 & 2.31 & 1.40 & 8.89
\end{tabular}

Epiandrum well developed but without membranous patches.

Male palp (Figs 12A-D, 13A-B): patella not modified or enlarged; tibia with ventral bunch of long setae, sharp dorsolateral apophysis pointing outward, and RTA, concavity between the two; RTA distally bifid with pointed inferior part and lamellar, truncated superior part; cymbium with basolateral flange and seven prolateral spines; subtegulum large and prominent, with three longitudinal ridges, posterior part swollen; tegulum with two parts, centre membranous; distal part with transverse groove and knob pointing back; embolus thin and whip-shaped; distal extremity in tegular groove.

\section{Female}

Unknown.

\section{Distribution}

Known from Mikembo Sanctuary near Lubumbashi in the Democratic Republic of the Congo (Fig. 16).

Palindroma sinis gen. et sp. nov. urn:Isid:zoobank.org:act:920F08EE-1F07-47FB-AE12-5124D27E7AB5

Figs $14 \mathrm{~A}-\mathrm{E}, 15 \mathrm{~A}-\mathrm{C}, 16$

\section{Diagnosis}

The male of $P$. sinis gen. et sp. nov. is easily recognized by the characters of the palp: the very simple RTA, which is a tiny protrusion, the short sharp, embolus and whitish setae on legs and abdomen.

\section{Etymology}

The species name is an arbitrary combination of letters forming a noun in apposition and containing 'sin' (Spanish for 'without') referring to the virtual absence of an RTA.

\section{Material examined}

\section{Holotype}

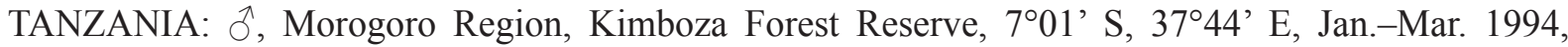
Frontier Tanzania (ZMUC).

\section{Paratypes}

TANZANIA: $2 \hat{\partial} \widehat{\partial}$, together with holotype (ZMUC); $2 \hat{\partial} \widehat{\partial}$, together with holotype (MRAC 244093). 
JOCQUÉ R. \& HENRARD A., Palindroma gen. nov., with novel zodariid synapomorphy

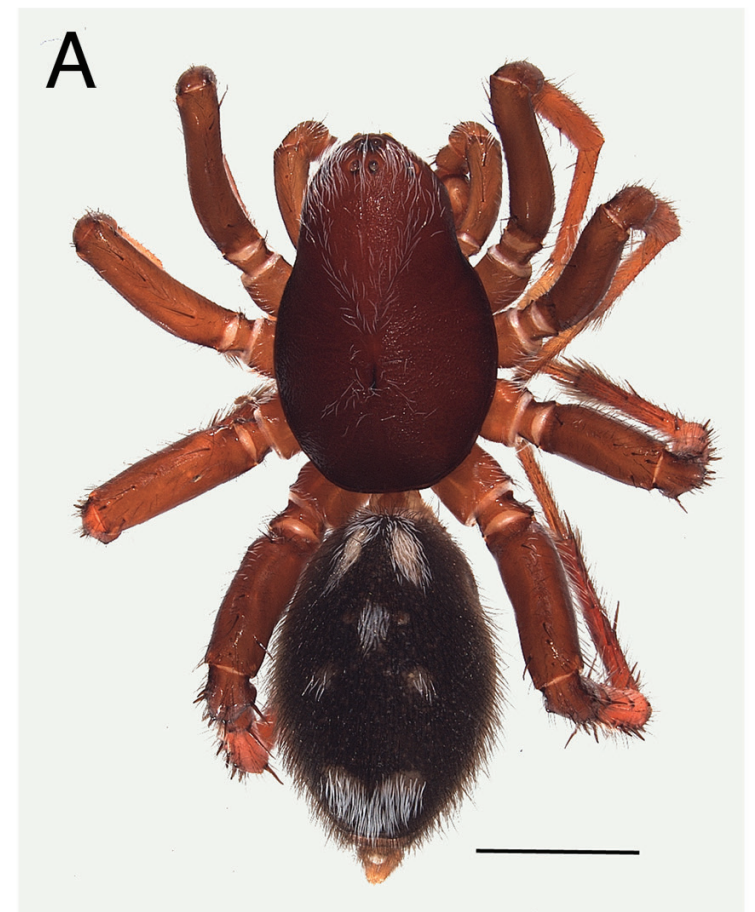

\section{B}
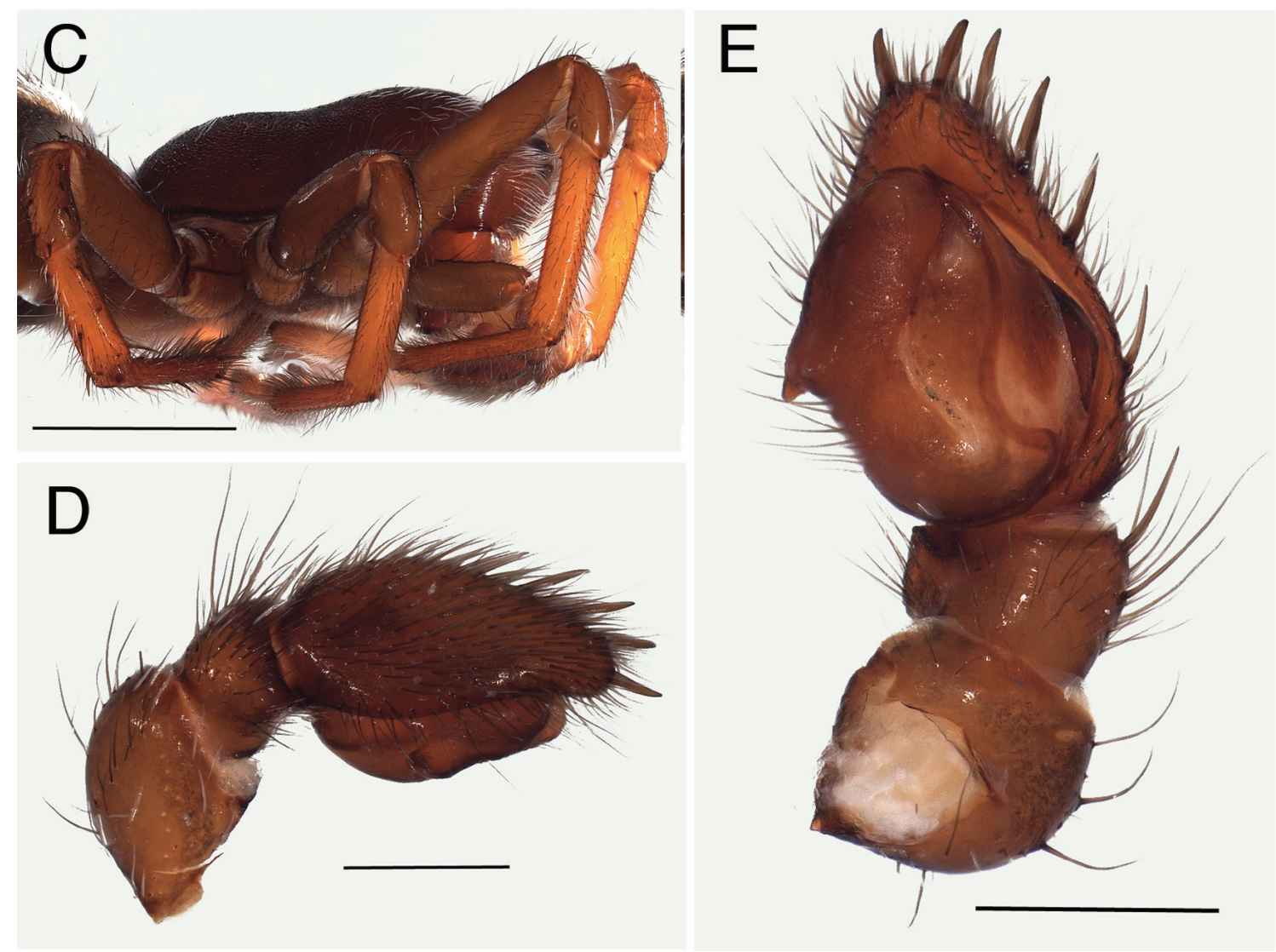

Fig. 14. Palindroma sinis gen. et sp. nov., holotype, $\hat{o}$. A. Habitus, dorsal view. B. Idem, ventral view. C. Idem, lateral view. D. Palp, retrolateral view. E. Idem, ventral view. Scale bars: A-C $=2 \mathrm{~mm}$, D-E $=0.5 \mathrm{~mm}$. 


\section{Other material}

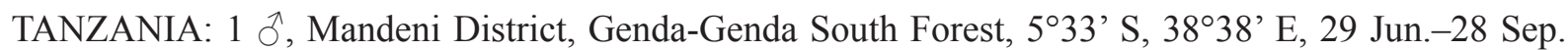
1991, Frontier Tanzania (ZMUC); 2 $\widehat{\jmath}$, Tanga District, Muneza Region, Magrotto Hill, $5^{\circ} 07^{\prime} \mathrm{S}$, 3845’ E, Jul.-Sep. 1994, Frontier Tanzania (ZMUC).

\section{Description}

Male (holotype)

Total length 8.38; carapace 4.19 long, 2.56 wide, narrowed to 1.49 in eye region.

Colour: carapace dark chestnut brown with silvery setae in eye region and around fovea (Fig. 14A-C); chelicerae, sternum and legs medium orange-brown; anterior tarsi yellow; sparse dorsal white hairs on all $\mathrm{T}$ and Mt and on $\mathrm{tI}$, dense on $\mathrm{t}$ I. Abdomen: dorsum dark grey with five small pale spots in anterior half, and large one in posterior half, all covered with white setae; four apodemes well developed; sides dark grey with sparse white hairs in anterior half; venter pale grey, well separated from sides by oblique pale band on either side; with two rows of apodemes; spinnerets yellowish brown.

Carapace finely reticulated, with conspicuous dip at level of fovea.

Eyes: AME: 0.13; ALE: 0.16; ALE-ALE: 0.20; AME-ALE: 0.15; PME: 0.13; PLE: 0.15; PME-PME: 0.10 ; PME-PLE: 0.30 . Clypeus 0.46 or 2.4 times width of ALE.

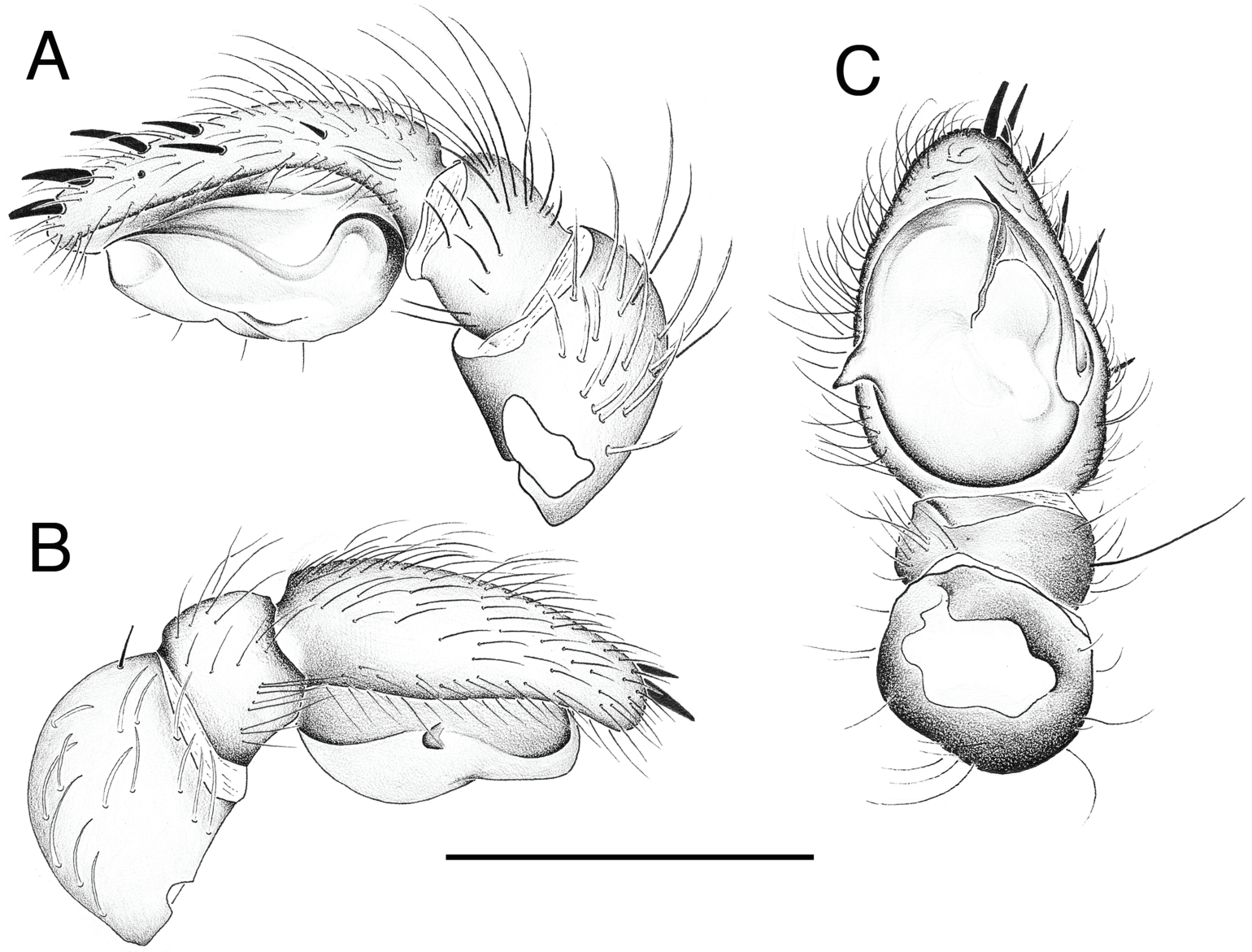

Fig. 15. Palindroma sinis gen. et sp. nov., holotype, ふ઼. A. Palp, prolateral view. B. Idem, retrolateral view. C. Idem, ventral view. Scale bar $=1 \mathrm{~mm}$. 
JOCQUÉ R. \& HENRARD A., Palindroma gen. nov., with novel zodariid synapomorphy

Chilum double, each sclerite 0.10 high, 0.25 wide; no setae. Chelicerae with distomesal membranous swelling. Sternum shield-shaped, 1.56 long, 1.35 wide.

Legs: anterior tarsi shorter than metatarsi.

Spination

\begin{tabular}{ccccc} 
& F & P & T & Mt \\
\hline I & pl1d2 & - & v1-1-1 & dw2 \\
II & pl1d2 & - & v1-1-1 & dw3 \\
III & d2rl1 & pl2d3rl1 & pl3d3rl1v2-2-2 & 4dispdw5 \\
IV & d2rl1 & pl2d3rl1 & pl3d5rl2v2-2-2 & 9dispdw5
\end{tabular}

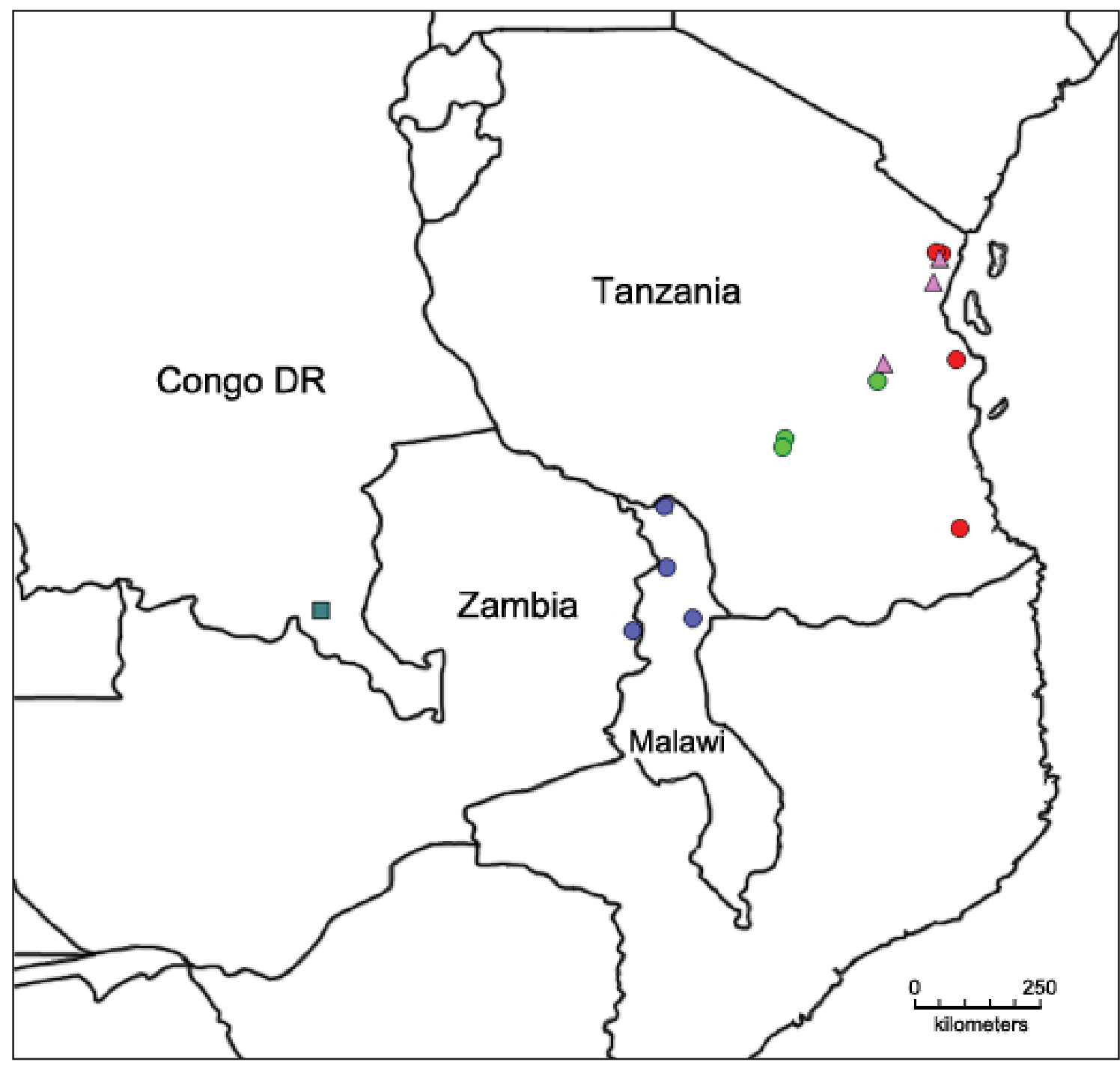

Fig. 16. Distribution map of P. avonova gen. et sp. nov.(๑), P. aleykyela gen. et sp. nov. (๑), P. morogorom gen. et sp. nov. (๑), P. obmoimiombo gen. et sp. nov. ( $\square)$ and P. sinis gen. et sp. nov. ( $\mathbf{\Delta})$. 


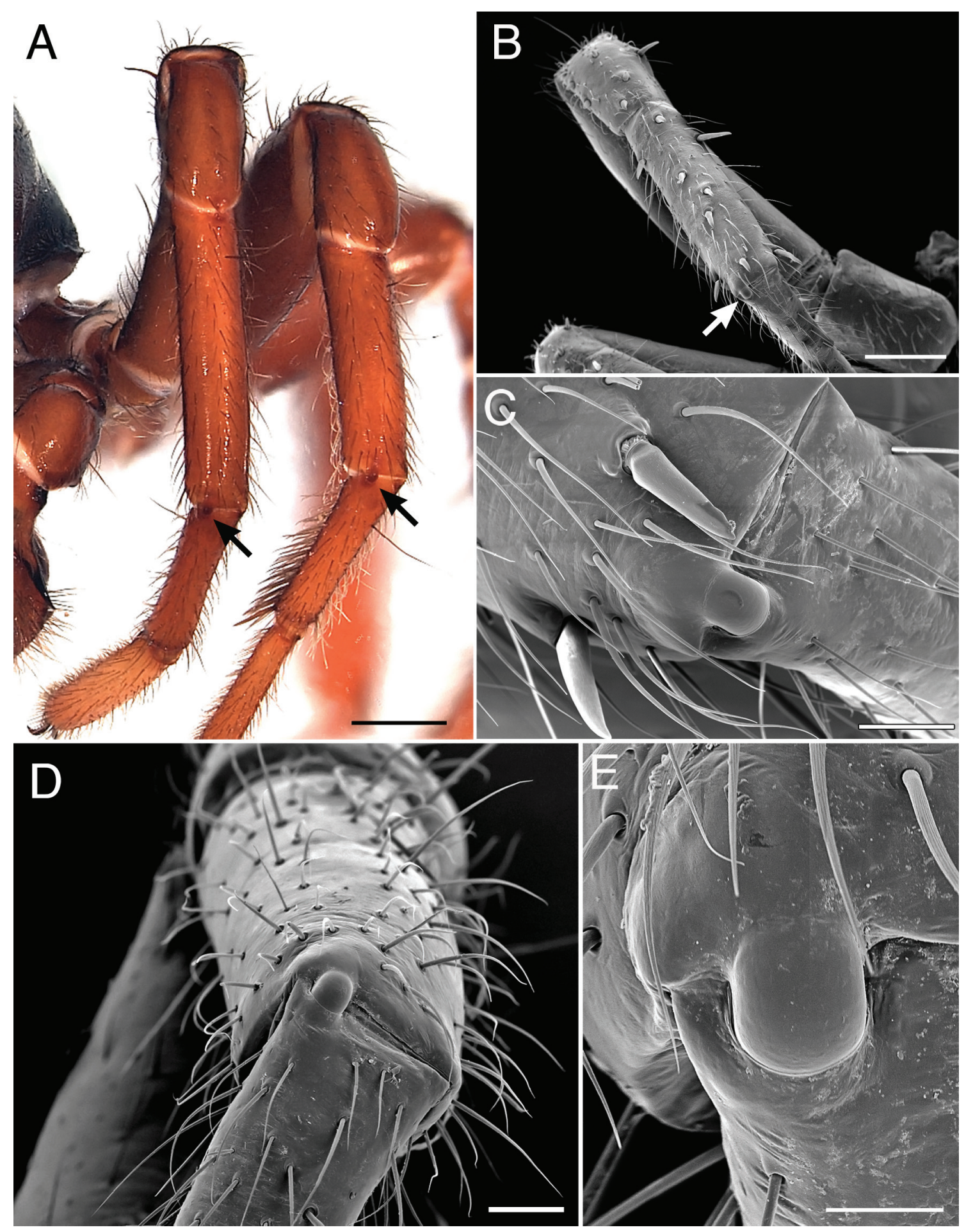

Fig. 17. Palindroma morogorom gen. et sp. nov. A. Left legs I and II, frontal view; arrows indicate tibial process. B. Left leg III, tibia and metatarsus, dorsal view. C. Detail of previous. D. Left leg II, tibia and metatarsus, dorsal view. E. Detail of previous. Scale bars: A-B $=0.5 \mathrm{~mm}, \mathrm{C}-\mathrm{D}=100 \mathrm{~mm}, \mathrm{E}=50 \mathrm{~mm}$. 
JOCQUÉ R. \& HENRARD A., Palindroma gen. nov., with novel zodariid synapomorphy

Leg measurements

\begin{tabular}{ccccccc} 
& F & P & T & Mt & t & Total \\
\hline I & 2.10 & 0.91 & 1.75 & 1.68 & 1.47 & 7.91 \\
II & 1.96 & 0.91 & 1.33 & 1.54 & 1.26 & 7.00 \\
III & 1.96 & 1.05 & 1.40 & 1.89 & 0.98 & 7.28 \\
IV & 2.38 & 1.12 & 1.96 & 2.73 & 1.33 & 9.52
\end{tabular}

Abdomen with well developed epiandrum; posterior margin with two semi-circular extensions, each preceded by tiny pit with membranous bottom.

Male palp (Figs 14D-E, 15A-C): RTA a tiny protrusion; tegulum with longitudinal cleft; prolateral part with sinuous sperm duct visible in transparency, retrolateral part with sharp, back-pointing knob; embolus short, straight, with sharp tip pointing forward. Without MA and conductor.

Female

Unknown.

\section{Distribution}

Known from Morogoro Region, Tanga District and Muheza District in Tanzania (Fig. 16).

\section{Discussion}

\section{The tibial process}

All representatives of Palindroma gen. nov. were found to have a characteristic tibial process (Fig. $17 \mathrm{~A}-\mathrm{D})$ on all legs of both sexes. It consists of a rounded lip-shaped extension of the distal, dorsolateral margin of the tibia, fitting in a shallow pouch on the proximal dorsal margin of the metatarsus. Scanning of other zodariids showed that the character appears to be present in all representatives of the family. It is obvious in almost all species, except in some taxa provided with a single femoral organ. In all observed species (see appendix) the process is situated near the prolateral side of the dorsal margin of the tibia (Fig. 17A). The relative size of the structure varies. It is particularly well developed in Palindroma gen. nov., but fairly small although still obvious in Cryptothele L. Koch, 1872 (Fig. 18C-D) as can be observed in fig. 2A of Ramirez et al. (2014). It is clearly visible in the other basal genera, for instance in Cyrioctea (Fig. 18E), Lachesana Strand, 1932 (Fig. 19A) and Cybaeodamus Mello-Leitão, 1938 (see Ramirez 2014: fig. 52e). It is conspicuous in representatives of the Storeninae: Amphiledorus Jocqué \& Bosmans, 2001 (Fig. 18A), Mallinella Strand, 1906 (Fig. 19B), Holasteron Baehr, 2004 (Fig. 18F). It is present and distinct in genera with a dual femoral organ: Asceua Thorell, 1887 (Fig. 18B), Suffasia Jocqué, 1991 and Suffrica Henrard \& Jocqué, 2015 (Fig. 19C), but has been overlooked by Henrard \& Jocque (2015). However, it is less conspicuous in the other taxa with a femoral organ where it is situated under the rim of the tibia and not on it as in the other taxa: Diores Simon, 1893 (Fig. 20E-F), Zodarion Walckenaer, 1826 (Fig.19D), Ranops Jocqué, 1991 (Fig. 19F). In some of the Zodariinae it is partly hidden and difficult to discern when the leg is not bent at the tibia-metatarsus joint: Microdiores Jocqué, 1987 (Fig. 19E), Parazodarion Ovtchinnikov, Ahmad \& Gurko, 2009 (Fig. 20C-D), Trygetus Simon, 1882 (Fig. 20A-B). The structure is absent in those taxa that have been regarded as putative sistergroups like Amaurobiidae (Fig. 21A), Agelenidae (Fig. 21B), Penestomidae (Fig. 21D), Corinnidae (Procopius sp., Cambalida sp.) Gnaphosidae (Haplodrassus sp.) or even in Titanoecidae (Fig. 21C). Another kind of tibial processes occurs in a few other families where a small process on both sides of the dorsal tibial rim is present. These have been called 'condyles' by Ramirez (2014: fig. 44b). However, they are obviously not homologous with the tibial process described here, since a tibial process and condyles may occur together on the rim of the tibia as in Cryptothele (Fig. 18C-D). The structure as found in Zodariidae appears to be a unique synapomorphy and its presence in basal zodariid taxa, 
e.g., Cyrioctea (Fig. 18E) and Lachesana (Fig. 19A), thus confirms the position of these genera in that family. This remarkable feature may be of great help for the identification of zodariid fossils or of spider leftovers containing a single intact leg, in vertebrate stomach contents for instance.
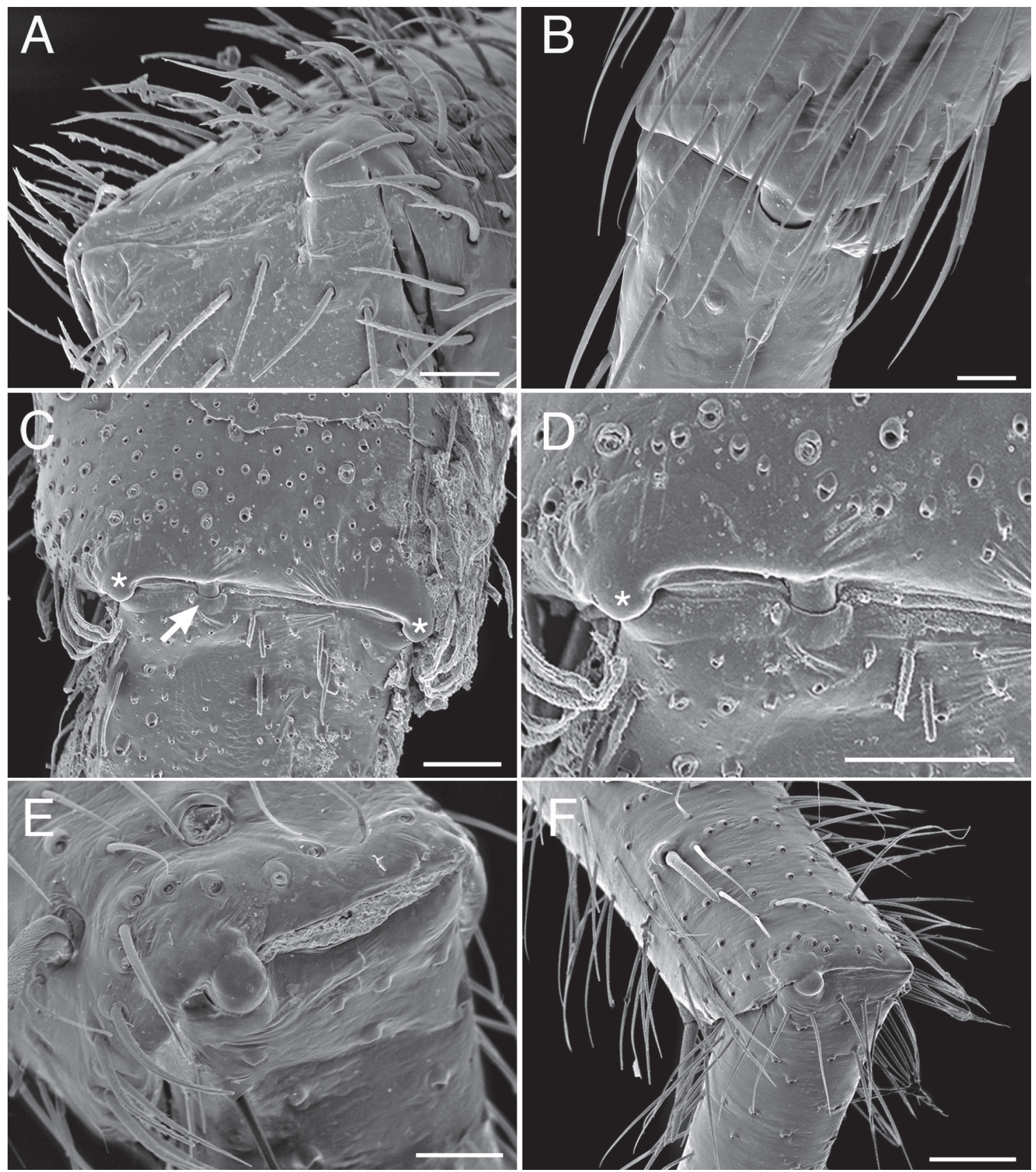

Fig. 18. A. Amphiledorus sp., juvenile, right tibia I. B. Asceua sp., ổ, right tibia and metatarsus IV. C. Cryptothele doreyana Simon, 1890, q, left tibia and metatarsus II. D. Idem, detail. E. Cyrioctea marken Platnick \& Jocqué, 1992, Ô, left tibia and metatarsus III. F. Holasteron aciculare Baehr, 2004, ${ }^{\lambda}$, left tibia and metatarsus III. Scale bars: A, E $=50 \mu \mathrm{m}, \mathrm{B}=20 \mu \mathrm{m}, \mathrm{C}-\mathrm{D}, \mathrm{F}=100 \mathrm{~mm}$. Arrow shows tibial process, asterisk indicates condyle. 
JOCQUÉ R. \& HENRARD A., Palindroma gen. nov., with novel zodariid synapomorphy

\section{Distribution}

In Jocqué (2009) and Jocqué et al. (2013) it was argued that the spider fauna of miombo woodlands has a character of its own and hardly overlaps with that of montane or coastal forests. The presently known distribution of the genus Palindroma gen. nov. is therefore somewhat puzzling. It is the first cryptotheline genus that is found in miombo woodland but it also occurs in coastal forest, even if
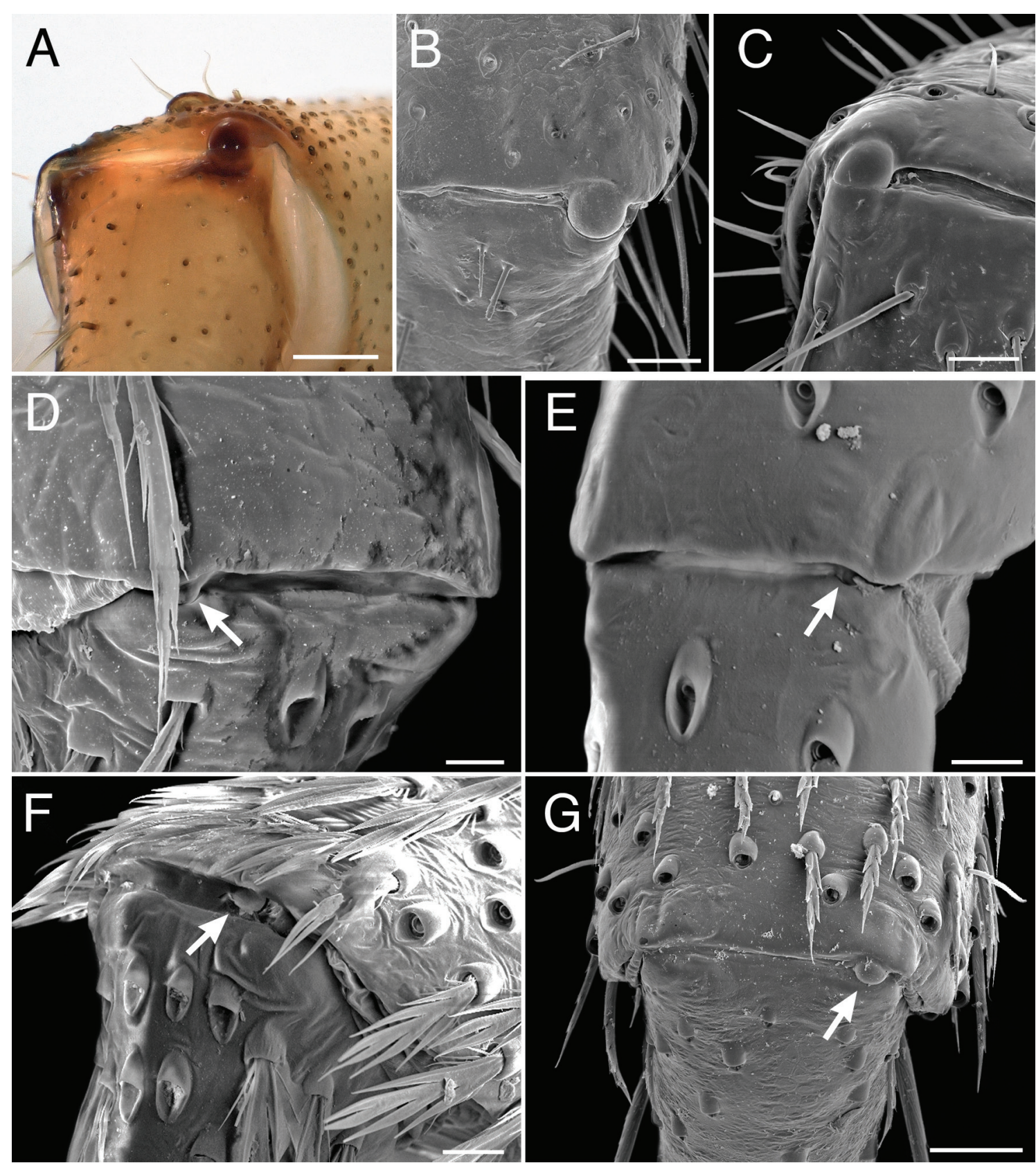

Fig. 19. A. Lachesana blackwalli (O. Pickard-Cambridge, 1872), $\widehat{\jmath}$, right tibia and metatarsus III, dorsal view. B. Mallinella sp., Ô, right tibia and metatarsus II. C. Suffrica gus Henrard \& Jocqué, 2015, ô, left tibia and metatarsus I. D. Zodarion nesiotes Denis, 1965, o, right tibia and metatarsus I. E. Microdiores sp., Ô, right tibia and metatarsus I. F. Ranops caprivi Jocqué, 1991, đ̂, right tibia and metatarsus II. G. Diores milloti Jocqué, 1990,, , right tibia and metatarsus I. Scale bars: A $=100 \mathrm{~mm}, \mathrm{~B}, \mathrm{G}=50 \mathrm{~mm}$, $\mathrm{C}, \mathrm{F}=20 \mathrm{~mm}, \mathrm{D}-\mathrm{E}=10 \mathrm{~mm}$. Arrow shows tibial process, asterisks indicate condyles. 
abstraction is made of $P$. obmoimiombo, which may belong to another genus as explained above. The vast miombo biome, 3.6 million $\mathrm{km}^{2}$ (against 2.3 million $\mathrm{km}^{2}$ for rainforest), is spread across eleven
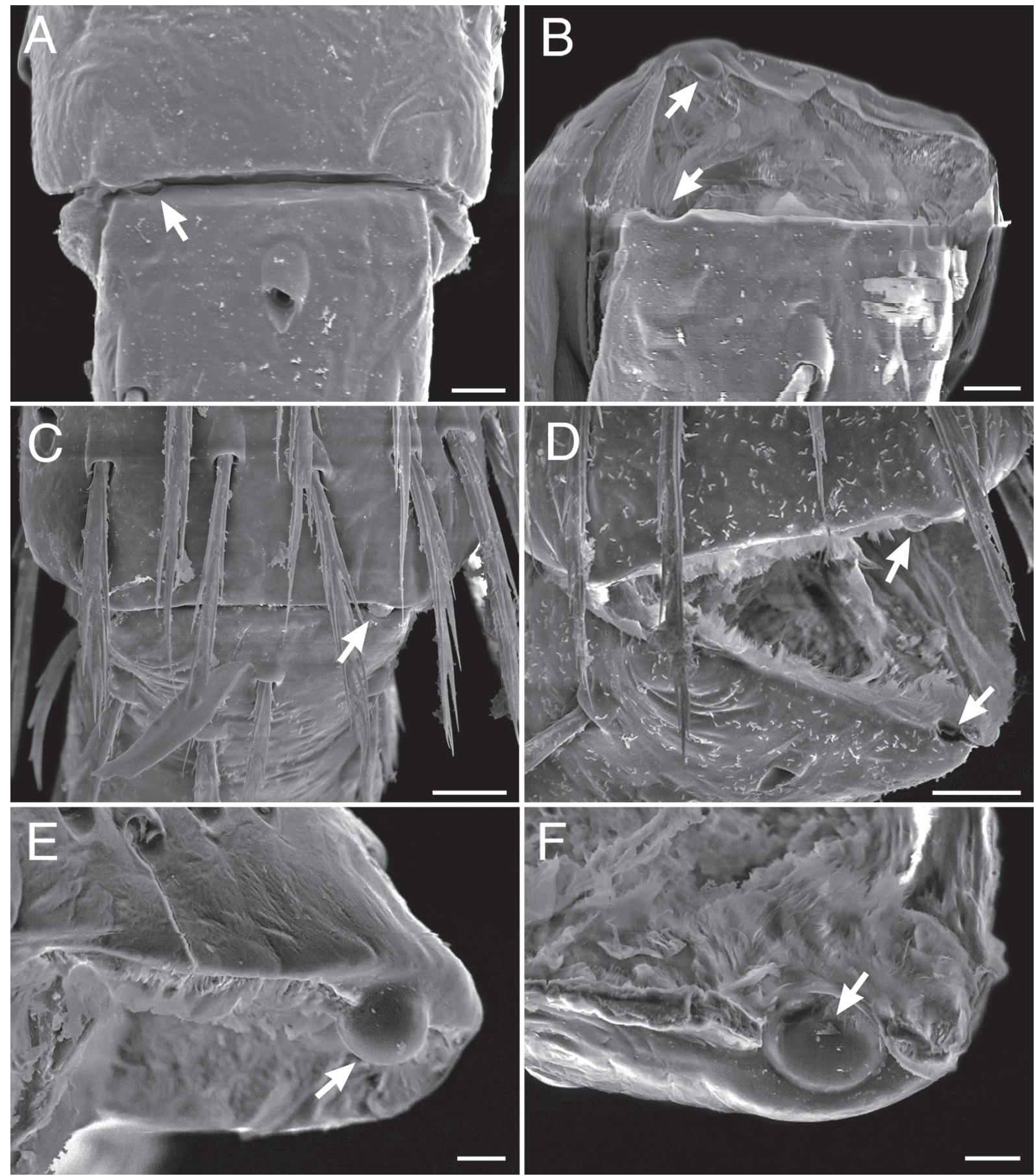

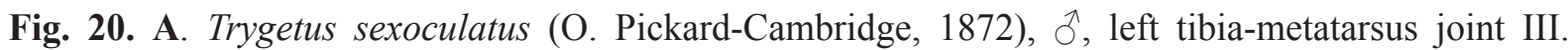
B. As previous, left tibia-metatarsus joint II; arrows indicate tibial process and metatarsal concavity. C. Parazodarion raddei (Simon, 1889), ô, right tibia-metatarsus joint III; arrow indicates tibial process. D. As previous, right tibia-metatarsus joint II; arrows indicate tibial process and metatarsal concavity. E. Diores poweri Tucker, 1920, 9 , right tibia-metatarsus joint IV; arrow indicates tibial process. F. Idem, detail; arrow indicates metatarsal concavity. Scale bars: A-B, E-F $=10 \mathrm{~mm}, \mathrm{C}-\mathrm{D}=20 \mathrm{~mm}$. 
JOCQUÉ R. \& HENRARD A., Palindroma gen. nov., with novel zodariid synapomorphy
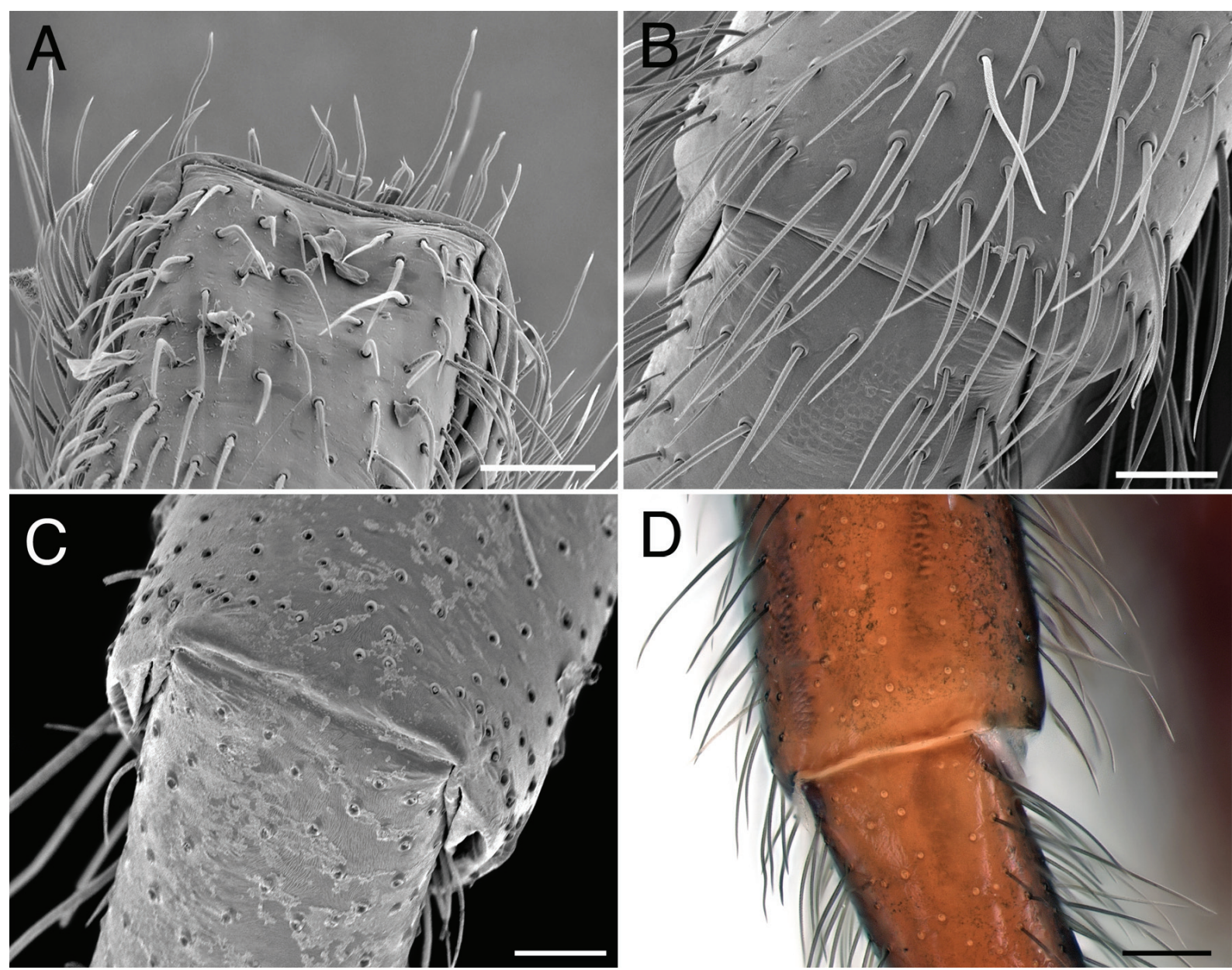

Fig. 21. A. Amaurobius fenestralis (Ström, 1768),, , base of right metatarsus 3. B. Coelotes terrestris (Wider, 1834), ภ, right tibia-metatarsus joint III. C. Nurscia albomaculata (Lucas, 1846), §, right tibia-metatarsus joint III. D. Penestomus montanus Miller, Griswold \& Haddad, 2010, $\odot$, right tibiametatarsus joint I. Scale bars: A-B, D $=100 \mathrm{~mm}, \mathrm{C}=50 \mathrm{~mm}$.

countries in southern Africa and is probably the least studied ecoregion of the continent. More detailed studies of the ecoregion will doubtlessly discover more species of the genus and most likely species that are closely related to $P$. obmoimiombo gen. et sp. nov.

\section{Acknowledgments}

We are grateful to Nikolaj Scharff (ZMUC) for the loan of material and to Alain Reygel for the splendid drawings. Additional material was provided by Barbara Baehr, Stefan Foord, Charles Griswold, Charles Haddad, Robert Raven and Milan M. Rézàc. The senior author has reached the age of 69 years, a perfect diagonal palindrome, and grabbed this occasion to create a genus of which the name was inspired by that term. This paper is publication BRC 340 of the Biodiversity Research Center (Universite Catholique de Louvain). This work was supported by the FRIA (Fonds pour la Formation à la Recherche dans l'Industrie et dans l'Agriculture). We thank Robert Bosmans and an anonymous referee for comments on the first draft. 


\section{References}

Henrard A. \& Jocqué R. 2015. On the new Afrotropical genus Suffrica with discovery of an abdominal gland and a dual femoral organ (Araneae, Zodariidae). Zootaxa 3972: 1-25. http://dx.doi.org/10.11646/ zootaxa.3972.1.1

Jocqué R. 1991. A generic revision of the spider family Zodariidae (Araneae). Bulletin of the American Museum of Natural History 201: 1-160.

Jocqué R. 2009. Some keep it short: on the radiation in the Afrotropical spider genera Capheris and Systenoplacis (Araneae, Zodariidae) without male pedipalp complexity increase. Journal of Afrotropical Zoology 5: 77-148.

Jocqué R.C.A.M. 2013. Cyrioctea (Araneae, Zodariidae) in Africa: temperate Gondwanaland relict, recent radiation, or both? European Journal of Taxonomy 47: 1-12. http://dx.doi.org/10.5852/ejt.2013.47

Jocqué R., Alderweireldt M. \& Dippenaar-Schoeman A. 2013. Biodiversity, an African perspective. In: Penney D. (ed.) Spider Research in the 21st century: 18-57. Siri Scientific Press, Rochdale.

Jocqué R. \& Dippenaar-Schoeman A.S. 2007. Spider Families of the World. Royal Museum for Central Africa, Tervuren.

Ramirez M.J. 2014. The morphology and phylogeny of dionychan spiders (Araneae: Araneomorphae). Bulletin of the American Museum of Natural History 390: 1-374. http://dx.doi.org/10.1206/821.1

Ramirez M.J., Grismado C.J., Labarque F.M., Izquierdo M.A., Ledford J.M., Miller J.A. Haddad C.R. \& Griswold C.E. 2014. The morphology and relationships of the walking mud spiders of the genus Cryptothele (Araneae: Zodariidae). Zoologischer Anzeiger 253: 382-393. http://dx.doi.org/10.1016/j. jcz.2014.03.002

Russell-Smith A. \& Jocqué R. 2015. New Zodariidae (Araneae) from Mkomazi Game Reserve, Tanzania. African Invertebrates 56: 455-476.

Manuscript received: 10 June 2015

Manuscript accepted: 8 September 2015

Published on: 13 November 2015

Topic editor: Koen Martens

Desk editor: Kristiaan Hoedemakers

Printed versions of all papers are also deposited in the libraries of the institutes that are members of the EJT consortium: Muséum National d'Histoire Naturelle, Paris, France; Botanic Garden Meise, Belgium; Royal Museum for Central Africa, Tervuren, Belgium; Natural History Museum, London, United Kingdom; Royal Belgian Institute of Natural Sciences, Brussels, Belgium; Natural History Museum of Denmark, Copenhagen, Denmark. 
JOCQUÉ R. \& HENRARD A., Palindroma gen. nov., with novel zodariid synapomorphy

\section{Appendix}

\section{Comparative material studied}

Amaurobius fenestralis (Ström, 1768) (Fig. 21A), \&, Belgium, Tervuren, under bark of tree, 1 Feb. 2011, hand catch, A. Henrard rec.

Amphiledorus sp. (Fig. 18A), sub adult $\widehat{\text { }}$, Portugal, Alentejo, 1 Apr. 2005, hand catch, M. Rézàc leg.

Asceua sp. (Fig. 18B), Ô, Phillipines, Luzon Island, Quezon Province, Mt. Banahaw de Luchan, 5.25 km WSW of Luchan, elev. 1279 m, $14^{\circ} 05.217^{\prime} \mathrm{N}, 121^{\circ} 31.036^{\prime}$ E, forest, 17-22 May 2011, propylene glycol pitfall traps, H. Wood, M. Yngente, N. Choussou Poludouri, C. Griswold \& V. Knutson rec., PH0029, CASENT-9042498.

Coelotes terrestris (Wider, 1834) (Fig. 21B), Ô, Belgium, Marchin, Porche de Roiseux, on litter near cliffs, 13 Sep. 2014, hand catch, A. Henrard rec.

Cryptothele doreyana Simon, 1890 (Fig. 18C-D), ㅇ, New Caledonia, Aoupinie top camp, 2111' S, $165^{\circ} 18^{\prime}$ E, 2-3 Nov. 2001, 850 m, sieved litter, berlesate 1060, G.B. Montheit (Queensland Museum QM90506).

Cyrioctea marken Platnick \& Jocqué, 1992 (Fig. 18E), đ̊, South Africa, Limpopo prov., 10 Jun. 2009, pitfall traps, S. Foord rec., 12N/B4.

Diores milloti Jocqué, 1990 (Fig. 19G), +, Madagascar, 17-20 Jan. 2009, general collecting day and night, CASENT 9031122.

Diores poweri Tucker, 1920 (Fig. 20E-F),, , South Africa, Free State Province, Bloemfontein, National Botanic Gardens, grassland, $29^{\circ} 02^{\prime}$ S, $26^{\circ} 12^{\prime}$ E, 28 Nov. 2012, Pitfall tarps, C. Haddad rec.

Holasteron aciculare Baehr, 2004 (Fig. 18F) (QM).

Lachesana blackwalli (O. Pickard-Cambridge, 1872) (Fig. 19A), Ô, Israel, Dead Sea, MRAC 169819.

Mallinella sp. Thailand (Fig. 19B) (ZMUC).

Microdiores sp. (Fig. 19E), $\widehat{\partial}$, Democratic Republic of the Congo, Mikembo, miombo woodland, Uapaca forest, 11²8' S, 027³9' E, 26 Oct. 2010, pitfall traps, R. Jocqué \& M. Hasson rec., MRAC 234392.

Nurscia albomaculata (Lucas, 1846) (Fig. 21C), ô, France, Adrets de l’Estérel, under stone, 17 Jul. 2013, hand catch, A. Henrard rec.

Parazodarion raddei (Simon, 1889) (Figs 20C-D), Ô, United Arab Emirates, Wadi Wurayah farm, 252ㄴ N, 56²17’ E, 13 Jan.-2 Mar. 2009, Malaise trap, A. Van Harten rec., MRAC 233874.

Penestomus montanus Miller, Griswold \& Haddad, 2010 (Fig. 21D), 9 paratype, Lesotho, Qachas Nek district, near Ha Mphahama, stone ridge, under rocks, 1820 m, 3005.520' S, 28³5.746’ E, active search, C. Haddad rec., SNCAP-2006/1535.

Ranops caprivi Jocqué, 1991 (Fig. 19F), Ô, South Africa, Free State Province, Bloemfontein, National Botanical Gardens, $29^{\circ} 02^{\prime}$ S, $26^{\circ} 12^{\prime}$ E, 12 Oct. 2012, C. Haddad leg.

Suffrica gus Henrard \& Jocqué, 2015 (Fig. 19C), đ’, Tanzania, Tanga, East Usambara Mts, Amani, 55.7’ S, $38^{\circ} 38^{\prime}$ E, 950 m, 28 Oct. -9 Nov. 1995, pitfalls, C. Griswold, N. Scharff \& D. Ubick leg., MRAC.

Trygetus sexoculatus (O. Pickard-Cambridge, 1872) (Fig. 20A-B), †, Egypt, Sinai, 50 km E of Suez, 31 Mar. 2000, M. Alderweireldt rec., MRAC 209667.

Zodarion nesiotes Denis, 1965 (Fig. 19D), , , Fuerteventura, near “La Pared”, under stones, 9 Apr. 2011, hand catch, A. Henrard leg. 